Portland State University

PDXScholar

\title{
A Qualitative Analysis for Sex Determination in Humans Utilizing Posterior and Medial Aspects of the Distal Humerus
}

Veronica L. Wanek

Portland State University

Follow this and additional works at: https://pdxscholar.library.pdx.edu/open_access_etds

Part of the Anatomy Commons, and the Biological and Physical Anthropology Commons Let us know how access to this document benefits you.

Recommended Citation

Wanek, Veronica L., "A Qualitative Analysis for Sex Determination in Humans Utilizing Posterior and Medial Aspects of the Distal Humerus" (2002). Dissertations and Theses. Paper 3571.

https://doi.org/10.15760/etd.5455

This Thesis is brought to you for free and open access. It has been accepted for inclusion in Dissertations and Theses by an authorized administrator of PDXScholar. Please contact us if we can make this document more accessible: pdxscholar@pdx.edu. 


\section{THESIS APPROVAL}

The abstract and thesis of Veronica L. Wanek for the Master of Arts in

Anthropology were presented September 24, 2002, and accepted by the thesis

committee and the department.

COMMITTEE APPROVALS:

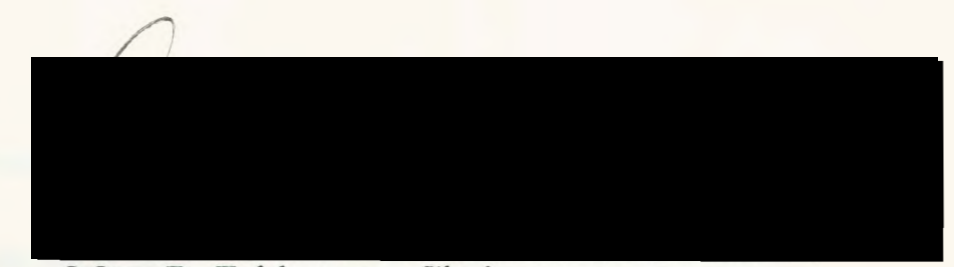

Marc R. Feldesman, Chair

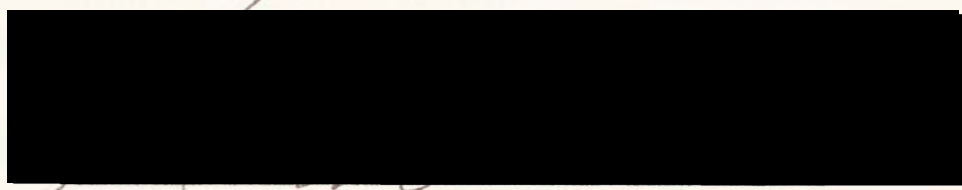

Kenneth Ames

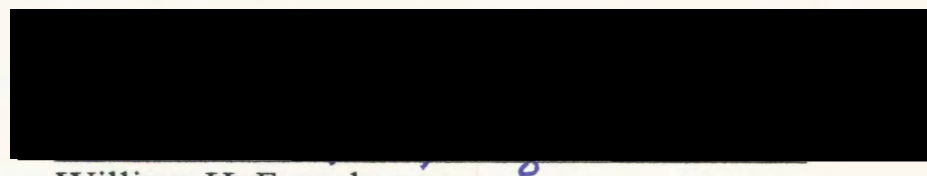

William H. Feyerherm

Representative of the Office of Graduate

Studies

DEPARTMENT APPROVAL:

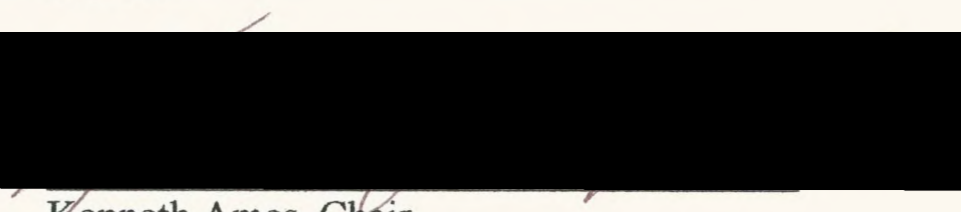
Kenneth Ames, Chair
Department of Anthropology 


\section{ABSTRACT}

An abstract of the thesis of Veronica L. Wanek for the Master of Arts in Anthropology presented September 24, 2002.

Title: A Qualitative Analysis for Sex Determination in Humans Utilizing Posterior and Medial Aspects of the Distal Humerus.

Visual and metric analysis both provide accepted methods for sex determination in humans. Visual ascertainment uses differing morphological traits in males and females to establish sex. Researchers have continually sought accurate methods of sexing long bones when skulls or pelves are absent or fragmented. These long bone elements may not have sexually distinct characteristics, but tend to survive in the field quite well.

Metric analysis depends on size dimorphism between males and females to correctly assign sex. Metric methods fail where the sexes overlap or when skeletal elements cannot be assigned to their correct biological population. Under these conditions, visual ascertainment is extremely useful. It relies on descriptive features, not size, to interpret the shape variations between male and female elements. For example, physiological soft tissue variations in the "carrying angle" of the arm at the humero-radioulnar junction are known to be sexually dimorphic; therefore, the hard tissue features of the distal humerus also should be sexually dimorphic. 
determine sex in a blind study conducted on 649 individuals sampled from diverse biological populations. In addition to visual assessment, I collected four humeral measurements to determine whether quantitative analysis would be a better indicator of sex than non-metric analysis. I used nonparametric statistical methods to examine the significance of each morphological feature and its relationship to known sex. All characteristics showed a high association with sex, and the relationships between sex and each characteristic were statistically strong. The final predictive quality of this method was $84 \%$ regardless of population; I concluded that my visual method is a dependable sex predictor among diverse populations. Every biological group varied considerably in size dimensions, but exhibited common morphological features of the distal humerus. This confirms that visual techniques provide accurate results regardless of biological affiliation. In many cases, visual assessment was as accurate or more accurate than metric analysis. Therefore, the distal humerus and its unique physical features provide an alternative method to previously used quantitative techniques in the determination of sex. 
A QUALITATIVE ANALYSIS FOR SEX DETERMINATION

IN HUMANS UTILIZING POSTERIOR AND MEDIAL ASPECTS

OF THE DISTAL HUMERUS

by

VERONICA L. WANEK

A thesis submitted in partial fulfillment of the requirements for the degree of

MASTER OF ARTS

in

ANTHROPOLOGY

Portland State University

2002 


\section{ACKNOWLEDGEMENTS}

Numerous individuals provided important contributions in the completion of this thesis. I'd like to thank Lyman Jellema and Lillian Rubin of the CMNH for their genuine interest and astute integrity in creating a truly blind study for me; Dr. David Hunt of the National Museum of Natural History for his assistance in navigating the Smithsonian and providing my diverse population sample; Dr. John Lundy, Head of Anthropology at Clark College (Vancouver, WA) and consulting Forensic Anthropologist for the Oregon State Medical Examiner's Office for his steadfast support and encouragement; Dr. Marc Feldesman, who is my advisor, Thesis Committee Chair and the Head of Anthropology at Portland State University, for his knowledge and gracious assistance in the improvement of this thesis; my research assistant, Ski Vance for her diligent and accurate compilation of my data (as well as being my mom); and my family and friends, especially my husband, Don, for their enthusiastic embrace of my decision to go to graduate school and continued, unfaltering support of my unique career choice. 
TABLE OF CONTENTS

PAGE

ACKNOWLEDGEMENTS..................................................

LIST OF TABLES................................................................ii

LIST OF FIGURES................................................................

CHAPTER

I INTRODUCTION.............................................

II METHODS AND MATERIALS.........................

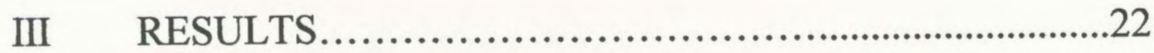

IV DISCUSSION ................................................ 46

$\mathrm{V} \quad$ CONCLUSION ...........................................54

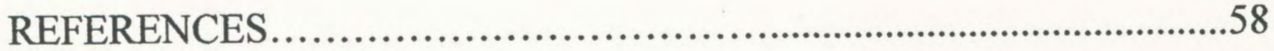

APPENDICES

A Modified Analytical Worksheet.......................60

B Collection Background, Lab Protocol, Documentation

And Observation Techniques...........................61 


\section{LIST OF TABLES}

PAGE

TABLE 1: Total sample size by population .34

TABLE 2: Relationship between variables and known sex..............35

TABLE 3: Accuracy of sex determination from features of the distal humerus, all populations represented.........................36

TABLE 4: Comparison of sex determination accuracy from the features

Of the distal humerus by population............................37

TABLE 5: Accuracy of sex determination from distal humerus features,

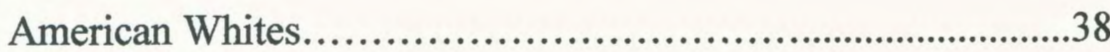

TABLE 6: Accuracy of sex determination from distal humerus features,

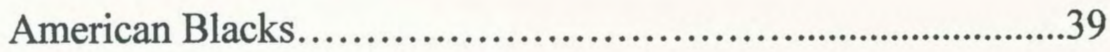

TABLE 7: Accuracy of sex determination from distal humerus features,

Native Americans. .40 


\section{LIST OF TABLES (CONTINUED)}

PAGE

TABLE 8: Accuracy of sex determination from distal humerus features,

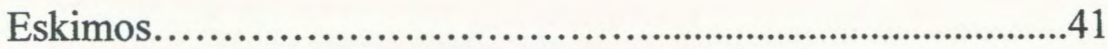

TABLE 9: Accuracy of sex determination from distal humerus features,

Aleutians

TABLE 10: Accuracy of sex determination from distal humerus features,

Chinese.

TABLE 11: Accuracy of sex determination from distal humerus features,

Japanese.

44

TABLE 12: Number of misclassified individuals with available

Measurements, by population. 


\section{LIST OF FIGURES}

PAGE

FIGURE 1: Trochlear Constriction.....................................19

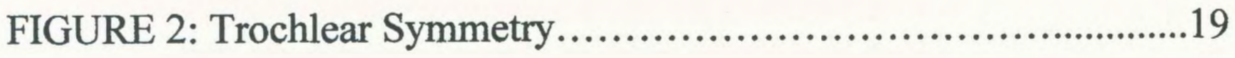

FIGURE 3: Olecranon Fossa Shape...................................19

FIGURE 4: Medial Epicondylar Angle..............................20

FIGURE 5: Medial Epicondylar Symmetry.......................20

FIGURE 6: Distal Spool Curvature ..................................20

FIGURE 7: Maximum Length of the Humerus . ......................21

FIGURE 8: Biepicondylar Width of the Distal Humerus................21

FIGURE 9: Transverse Diameter and Vertical Diameter of the

Humeral Head ...........................................................21 


\section{Chapter I: Introduction}

Visual characteristics and osteometry provide accepted methods for sex determination of unidentified skeletal remains. Qualitative (visual) determination is the use of morphological traits that establish whether a skeletal element is female or male, due to sexual dimorphism. For example, when researchers recover remains that include an intact cranium and pelvis, qualitative morphological interpretation is quite accurate for assigning a specific sex to the remains (Holman, 1991). Quantitative (metric) analysis uses skeletal measurements from a known population to provide the same answer; in this case, the sex of the individual element examined. Combined, the use of quantitative and qualitative analysis plays a significant role in determining skeletal sex. When metric analysis is used exclusively, however, problematic circumstances may arise. The lack of grossly distinct sexual dimorphism in human limbs can make qualitative analysis challenging. To compound the problem, fragmentary limb remains are difficult to measure. Therefore, researchers have continually sought accurate methods of sexing surviving skeletal elements when the pelvis and cranium are absent. These methods are particularly desirable for long bones, which may not have sexually distinct characteristics, but tend to survive in the field quite well. Thus, the pursuit of sexually dimorphic characteristics on long bone elements such as the humerus, ulna, radius, femur, tibia and fibula goes on. So far, the results are equivocal.

Sex determination of post-cranial elements has been studied extensively. 
Very early on, researchers used quantitative analysis and discovered its benefits; however, most agreed that nothing could take the place of a visual assessment of specific skeletal element characteristics by a trained and experienced analyst. Although some researchers considered measurements to be more accurate than visual techniques, they argued continually that there are inherent limitations to measurements, “...namely, that they are poor descriptive agents and are subject to various kinds of error. As is all too well known to anthropometrics, an index tells nothing but the percental relationship of two linear dimensions; it tells nothing about the shapes of the parts included within the dimensions." (Stewart, 1954, p. 390)

As new sex determination techniques emerged for both visual and metric analyses, so did conflicts over the accuracy and applicability of each method. Specifically, the debate has centered around studies advocating quantitative techniques. Poor performance of visual indicators eventually led to a greater emphasis on measurements. Visual assessment of morphology was left to experts, but considered not as objective as numerical formulae applied by even the most inexperienced examiner. Researchers believed quantitative examinations employed unbiased procedures because they dealt with numerical values; visual assessment was subject to judgment errors by the researcher attempting a specific technique. However, similar issues arose in metric studies of long bones, as exemplified by the prediction errors made with certain contemporary "canonical" formulae for tibia research (Jantz et al, 1994). This indicates quantitative methods 
were and continue to be more subjective than once thought. Nonetheless, as quantitative analysis ultimately became the norm for predicting stature, it also became the norm, by default, for sex determination as well.

Some metric techniques have proven to be accurate sex predictors when used on certain skeletal elements (Mall et al, 2001). For example, long bones appear ideal for quantitative sexing methods because most exhibit clear and discernible differences in size dimensions, but seem to lack visible physical indicators of sex (King et al, 1998). Specific measurements taken from the humerus correctly predict sex between $64 \%$ and $80 \%$ of the time if the most accurate measurement suite is used. However, this accuracy also illustrates that considerable variation between male and female humeri still exists. For example, the lengths of male and female black humeri overlap considerably in one analysis, causing misassignments of sex. In the same analysis, however, white male and female humeral length are bimodally distributed and assignment of sex is quite accurate (see Holman and Bennett, 1991). In addition, several researchers report accurate sex determination when measuring the transverse and vertical proximal head of the humerus, but accuracy decreases when using measurements of the distal articular region of the same bone (France 1988, van Dongen 1963). In all studies to date, distal humerus measurements were less reliable in separating sexes in each population than were the proximal measurements. Therefore, the accuracy in humeral metric sexing techniques is dependent on the humeral dimensions used; humeral metrics are population-dependent; and populations have noticeably 
diverse size distributions. In fact, the dominant factor in quantitative analysis for all populations is nearly always the size distribution of males and females.

However, when size is "removed" from the statistical analysis of sex, what is left to analyze? Since men are morphologically larger than women are (a nonpopulation-specific trait), quantitative analysis depends almost completely on these size differences to correctly assign sex. This method fails where the sexes overlap (i.e., a large female or a small male). In addition, different biological populations have grossly different size distributions. Comparative data on humerus lengths among whites, American Indians, American blacks and Australian aborigines show wide variation in size dimensions; American Indians are the smallest represented, and the American blacks are the largest (van Dongen, 1963). If skeletal elements cannot be assigned to a population, how can accurate results be obtained from applying population-specific quantitative techniques? Under these conditions, non-metric analysis is extremely useful. Visual assessment offers an alternative that relies on descriptive features and observer experience to interpret the distinct variation in shapes between male and female elements, and to come ultimately to an accurate conclusion.

When proven sex-specific elements such as the pelvis and skull are missing or fragmented, the analyst must utilize other elements. Physiological soft tissue variations in the "carrying angle" of the arm at the humero-radioulnar junction are known to be sexually dimorphic; therefore, it follows that the hard 
tissue features of the distal humerus should be sexually dimorphic as well ${ }^{1}$ (Rogers, 1999). I have examined the effectiveness of a unique visual method of sex determination by observing and documenting specific non-metric skeletal features of the posterior and medial distal humerus. I hypothesize that, given a specific suite of characteristics, this method will be at least as accurate as known metric techniques (between 64\% and 80\%) in determining the sex of an individual. This technique does not rely on size, but on certain well-recognized, observable traits. If my hypothesis is correct, it would provide a new means to determine the sex of unidentified skeletal remains found in archaeological and contemporary settings. It very well may provide a more rapid determination than metric techniques. In addition, through comparative study of large modern human skeletal samples of mixed sex and ethnicity, I expect to be able to make inferences about 1) the reliability of these methods for different biological populations, 2) whether variations between biological populations are significant enough to note and great enough to make distinctions, 3) where the method tends to break down or misassign the sex of the skeletal element, and whether the misspecification is population specific, and finally 4) the presence of alternative features that might compensate for inaccuracies and increase sexing precision for a particular

\footnotetext{
'The "carrying angle" of the arm refers to the lateral deviation or "pitch" of the human forearm from the distal humeral axis. Ligaments connecting the distal humerus at the radioulnar joint provide a different range of extensor movement from males to females. In addition, the screw-like axis of the same joint creates dissimilar flexor alignment of males and females. This culminates in an approximate 10 to 15 degree carrying angle in males and a 20 to 25 degree carrying angle in females (Grabiner, 1989).
} 
population. Given the accuracy of some existing methods of metric sex determination, it is my hope that this technique would provide at least $80 \%$ accuracy to be considered useful as an alternative or complementary method. 


\section{Chapter II: Methods and Materials}

In 1999, Tracy L. Rogers of the Department of Anthropology at the University of Toronto performed a sex determination study using qualitative (nonmetric) visual traits from the distal humerus of European specimens (Rogers, 1999). Her analysis used trochlear constriction (Fig. 1), trochlear symmetry (Fig. 2), olecranon fossa shape and depth (Fig. 3), and the angle of the medial epicondyle (Fig. 4) as sex defining characteristics. These features combined to yield an $88.6 \%$ accuracy rate when applied by a trained observer. This compared to quantitative techniques that only yielded a $64 \%$ accuracy rate (Dittrick and Suchey, 1986). Rogers' paper initially laid the groundwork for visualization of certain sex-specific traits of the posterior distal humerus. My interest in testing the accuracy of sex determination by this method inspired me to examine the method more closely. I conducted two subsequent evaluations on independent small collections of humeri to determine the relative accuracy of the four traits. In the first trial (Spring 2000), I used Rogers' method with a 79\% accuracy rate when applied to an extremely small population of Caucasians, African Americans and Asians taken from the Oregon State Medical Examiner's Office. I applied the method a second time to a small skeletal collection of known sex from Portland State University two years later. I achieved a $78 \%$ accuracy rate with all features combined. This second trial provided a critical glimpse into limitations of the original characteristics. When isolated from each other, certain features tended to perform better than did others. Trochlear symmetry and the angle of the medial 
epicondyle easily separated males and females, and predicted sex at a high degree of accuracy ( $92 \%$ and $96 \%$, respectively). Olecranon fossa shape, depth, and trochlear constriction were less reliable. In addition, females exhibited more male morphology than males exhibited female morphology, resulting in the misidentification of three female humeri as male.

I revised the original feature descriptions and added two new features, both from the medial aspect of the distal humerus. These new features appeared to be complementary and supplemented the first four characteristics. Below are complete definitions of the final six features utilized as well as the visual procedure I used in this thesis. They are listed in the order I observed them on each specimen.

Trochlear constriction (Fig. 1): In males, the trochlea has a less constricted, "weaker" medial portion when observing the posterior surface of the distal humerus. In females, the trochlea exhibits a constricted spool shape and pronounced compression. Robusticity and an overall subtleness to the constriction depict male morphology; dramatic flexion and gracility depict female morphology ${ }^{2}$. I observed this trait from the posterior aspect at a ninety-degree angle.

${ }^{2}$ In describing what each characteristic may be visualized as, it should be mentioned that the description given is of the most distinct, unambiguous and easily observed features for males and females. These descriptions represent the ideal. 
Trochlear symmetry (Fig. 2): In males, the medial edge of the trochlea extends

further distally than does the lateral edge, making the trochlea appear asymmetrical or lop-sided. The distal extension of the medial and lateral edges of the female trochlea is almost equal. In obvious male humeri, the medial edge of the trochlea observably extends past the lateral edge. Female humeri, on the other hand, tend to be more symmetrical in shape. The trochlea, however, does extend past the lateral edge in all cases, male and female; female humeri then can only be categorized as almost symmetrical with this study, and not truly symmetrical. This feature is observed in the same position as trochlear constriction.

Olecranon fossa shape (Fig. 3): In males, the fossa appears roughly triangular; in females, the fossa appears oval. Subtleties in shape will exist. A distinct proximal extension, as prominent as the fossa itself and coming to a significant point depict male morphology. No proximal extension and no observable points of convergence to form a triangular shape depict female morphology ${ }^{3}$. A proximal extension, rising towards the proximal shaft and ending in a rounded margin from a distinctly oval olecranon fossa was considered a female trait as well. While observing feature trochlear symmetry, I could without difficulty analyze olecranon fossa shape.

\footnotetext{
${ }^{3}$ Rogers' paper originally implemented a depth feature as well as shape. Ambiguity existed in determining what was actually deep and what was shallow; therefore, the depth of the olecranon fossa was not used as a defining characteristic. I only implemented shape as a criterion.
} 
Angle of the medial epicondyle (Fig. 4): The angle at which the medial

epicondyle extended away from the spool of the trochlea was the basis for feature number four, the angle of the medial epicondyle. In males, the medial epicondyle extends parallel to the table or exhibits a slight angle when placed posterior side up and viewed from the distal end. The female medial epicondyle clearly angles upward, away from the plane of the tabletop surface when viewed from the same perspective. As the worksheet (Appendix A) illustrated, a slightly raised angle was considered male. Only a very dramatic elevation of the medial epicondylar angle was considered a female trait.

Medial epicondylar symmetry (Fig. 5): This feature describes how the medial epicondyle was positioned in relation to the circular outline of the trochlea. This feature appears on the medial aspect of the distal humeral element. In males, the medial epicondyle sits more centrally within the circular profile of the trochlea with the lateral surface of the bone placed on the table. In females, the medial epicondyle sits more towards the posterior edge of the trochlea. Anterior asymmetry (a more broad trochlear surface anteriorly) was considered a female trait, and equality of the trochlear surface on both the anterior and posterior sides surrounding the medial epicondyle was considered a male trait.

Distal spool curvature (Fig. 6): The humeral element was maintained in the same position for assessing medial epicondylar symmetry to assess distal spool 
curvature. In males, the distal spool profile is more perpendicular to the tabletop when the lateral surface of the bone is placed on the table. In females, the distal spool exhibits more of a curved profile when viewed from the same perspective. When observing this feature, the analyst must determine the overall degree of curvature seen in relation to the flat tabletop itself.

The actual observation phase was quite simple. I observed each bone individually, without the aid of initial measurements, photographs of "known" characteristics pertaining to one sex or the other as a reference, or any other implement that might bias my observation of the distinct individual element I examined. I relied on a worksheet that I developed (and subsequently modified) that described briefly each feature, and numbered them "\#1" through "\#6", corresponding to the sequence of the six features described above (Appendix A). A duplicate log was also used to record data. The sex characteristic was logged as either male ("M") or female ("F"). I observed each characteristic independent of the others, or at least I made the effort to do so in every case. I disregarded the proximal head and shaft during the initial examination of the distal portion. All features on the humerus were observed from the posterior and medial sides.

I observed and analyzed only left humeri. The reasoning behind this decision was based specifically on the collection demographics. Most of the Hamann-Todd and the Terry Collection specimens were originally collected and curated during a period when culture influenced handedness. During this time (1920-1959), left-handed children were forcibly shifted to right-handedness by the 
school system as well as by their parents. Left-handedness was seen as a type of peculiar abnormality and was actively discouraged. Consequently, the preponderance of specimens was most likely from right-handed individuals; hence the left humerus would be less affected by occupational stress (France, 1988). I considered left humeri to be a pragmatic and logical choice that would not obscure my effort to base conclusions on shape, and not size or occupational/ activity patterns.

In addition to visual assessment, I collected four commonly used humeral measurements (France, 1988) on a number of samples to provide a baseline of sex determination accuracy utilizing both methods. I chose the specific measurements based on their relationship to the distal humerus and their potential for accurate sex determination (France, 1988). The maximum length (Fig. 7) of the humerus was measured in centimeters with an osteometric board and consisted of the greatest length spanning from the most superior portion of the humeral head to the most distal portion of the trochlea. I chose this measurement because it represented a distinct size criterion, something I wanted to eliminate. The second measurement collected was biepicondylar width (Fig. 8), the distance between the most medial point on the medial epicondyle and the most lateral point on the lateral epicondyle (Hamilton, 1982 as referenced in France 1988), taken in millimeters with digital metric calipers. This measurement was chosen based on its obvious and direct correlation to my own distal humerus analysis, and is also (as are all measurements) based on the size of the element. The third and fourth 
measurements were the transverse and vertical diameters of the humeral head (Fig. 9a and 9b). The transverse diameter was taken across the humeral head on the articular surface, perpendicular to the shaft. The vertical diameter of the humeral head was measured parallel to the long axis of the humeral shaft, on the articular surface once again. I obtained both with digital metric calipers, and recorded them in millimeters.

There were two reasons for collecting measurements while doing a qualitative analysis. First, I wanted to take selected measurements to determine whether quantitative analysis would be a consistently better indicator of sex than the qualitative analysis I was performing. I took the complete suite of measurements on roughly one out of every ten humeral elements observed at the Cleveland Museum of Natural History and the Smithsonian. In addition, when I found some humeri to be visually ambiguous, I measured them to determine if quantitative techniques could sex an individual accurately where visual characteristics could not. I measured all humeri considered too ambiguous to accurately sex with qualitative analysis, to determine whether quantitative analysis would provide more accurate results (France 1988, Bass 1995). I used two metric studies as baselines for the quantitative techniques. France's technique (Bass, 1995) required the insertion of single variables into several regression formulae. The formulae supply a "cutoff" number, and sex was assigned based on whether the outcome was above or below the cutoff. Stewart's quantitative procedure (Bass 1995) required the comparison of the vertical diameter of the humeral head 
measurement to a "cutoff" value as well. If the measurement fell below a certain value, it was considered female, while it was considered male if it exceeded the cutoff. Any measurement that fell within an intermediate size range was considered indeterminate.

I utilized samples from the Hamann-Todd Collection housed at the Cleveland Museum of Natural History, and samples from the Terry Collection located in the National Museum of Natural History (Smithsonian) in Washington, D.C. for the formal research and analysis. Both collections housed mostly American whites and blacks, but they also curated specimens from other populations, which I used as well. Incorporating diverse population samples in testing the method was essential to determining whether the sex-specific features of this method were also population-specific.

The Cleveland Museum of Natural History (CMNH) on the campus of Case Western Reserve University in Cleveland, Ohio houses the Hamann-Todd Human Skeletal Collection, one of the more extensive skeletal assemblages of American blacks and whites in the United States. All 3,100 of the individuals curated in the Hamann-Todd Collection are catalogued and include age at death (estimated if not known), height, race, sex, date of birth, and approximate date of death. Records kept on the cadavers include death certificates, clinical histories, and anatomical reports of past injuries or pathology if available. The HamannTodd Collection is essentially "the unclaimed dead of Cleveland", recovered by Dr. T. Wingate Todd from the Case Western Medical School between 
approximately 1920 and 1960 . The majority of individuals represented are foreign-born whites (European) and native or foreign-born Negroes (Cobb, 1932). Appendix B provides additional information and background on the specific collections I observed.

I also considered other smaller populations curated at the $\mathrm{CMNH}$ for their utility in determining whether or not the sex determination method attempted was non-population-specific. I observed one Egyptian, one Australian Aborigine, and two native Hawaiians in the study. Most of the Native American remains that previously were curated at the CMNH have now been successfully repatriated to their designated tribe of origin. I considered a small number of Native American specimens of unknown tribal affiliation to provide information, however small, on the non-population-specific capabilities of this method.

I observed over 300 humeri in the Hamann-Todd Collection, and with the assistance of those at the $\mathrm{CMNH}$, I was able to produce a purely blind study sample to test this analytical method. The bones have only an identification number permanently written on the shaft of the bone; there are no other distinguishing markings on any surface of the humeri. In no way was it obvious how the individual was categorized by observing the log number on the element. The collection is curated randomly with respect to its numbering system; there was absolutely no way to determine from the log number what the sex and race of the individual could have been.

Approximately three weeks separated my analysis of the Hamann-Todd 
Collection and my analysis of the curated skeletal collections, including the Terry Collection, at the National Museum of Natural History (Smithsonian) in Washington, D.C. Beginning in the 1920's, Dr. Robert J. Terry, a professor of anatomy and head of the Anatomy Department at Washington University Medical School in St. Louis, Missouri began to collect human skeletons from cadavers used in the Medical School's anatomy classes (Hunt, pers.comm.). The Terry Collection, as it exists now, consists of 1,728 specimens of known age, sex, ethnic origin, cause of death and pathological conditions. Records on the individuals curated include anthropometric measurements, dental charts, bone inventories and autopsy reports. The overwhelming majority of the sample were American blacks and whites; however, multiple populations were highly accessible and did have accurate sex designation assigned to them as well. I was able to retrieve data on Alaskan Aleutians, Chinese, a small sample of Japanese, Australian Aborigines, an Arikara Indian population from South Dakota, Basutu, one Jamaican, one Puerto Rican, one Patagonian, one Chilean, and a large Alaskan Eskimo population.

Several challenges arose while observing the Smithsonian material. The Alaskan Aleutian population was large, but displayed the sex of the individual in writing on the humeral shaft of the bone. I employed a method of "blinding" myself to this information to reduce the obvious bias. Ultimately, I believe most (if not all) bias was removed by the sheer quantity of humeri that I examined at one time. As at the Cleveland Museum of Natural History, The Smithsonian's Terry Collection is curated randomly. The bones have only an identification 
number permanently written on the shaft of the bone; there are no other distinguishing markings on any surface of the humeri. It was impossible to determine the sex and race of the individual from the log number. Furthermore, after collecting a sample of thirty to forty bones from the Terry Collection and returning to perform the analysis, I had no way of remembering what was a white male or a black female. With these precautions, I feel I achieved sufficient "blinding" for the majority of the sample taken at the Smithsonian, even though I was in charge of sampling from each of the several collections I had access to. I procured and analyzed the humeri in the same manner as at the CMNH. I applied the same techniques used in that trial for continuity, and I used the same worksheet (with one modification) originally developed. In addition to completing the worksheet for each individual, I maintained a duplicate log as well. I observed each characteristic independent of the others, or at least I made an effort to do so in every case.

I used nonparametric statistical methods (Pearson's chi-square tests, Spearman R, Kendall Tau) to examine associations between known sex and estimated sex based on each characteristic, and to gauge the significance of each morphological feature and its relationship to the others. When employing Pearson's chi-square tests, I determined the independence of each variable to the actual sex of the specimen. The chi-square value is based on the sum of the differences between observed and expected values for each category (in this case, morphological features determining sex and the known sex of the specimen). I 
performed these calculations with the entire sample, and then divided the sample into their biological affiliations in order to observe any unusual statistical anomalies that might exist in a specific group. When I used the Spearman R and Kendall Tau tests, both yielded identical results as the chi-square tests. I used the Windows 1.5.1 version of the open source statistical package called $\underline{R}$ (Ihaka and Gentleman, 1996). Specific functions used included cor.test(), chisq.test( ), table(), xtab(), str( ), and summary( ). To study each group separately, I used R's powerful subsetting functions illustrated as follows:

$>$ americanblacks $<$-humerusdata[humerusdata[,10] $==$ ="b",

$>$ americanwhites $<-$ humerusdata[humerusdata $[, 10]==" w "]$

If I needed to exclude only American blacks from the complete data set, the following function was used:

>humerusdatanoblacks<-humerusdata[humerusdata[,10]!="b"] 


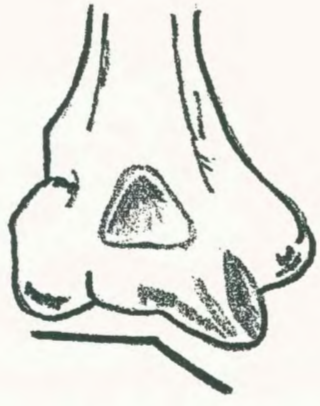

Male

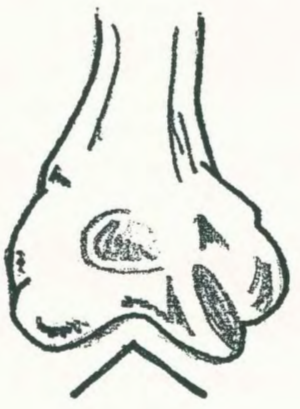

Female

FIG 1: Trochlear Constriction- in males, the trochlear constriction is weak, in females it is more constricted and "spool-shaped".

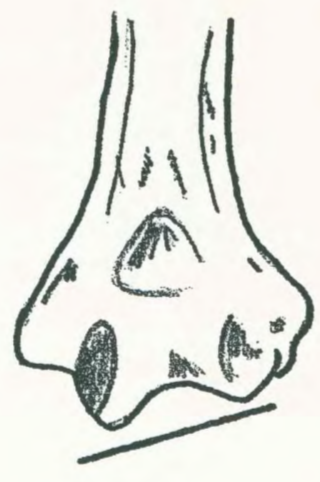

Male

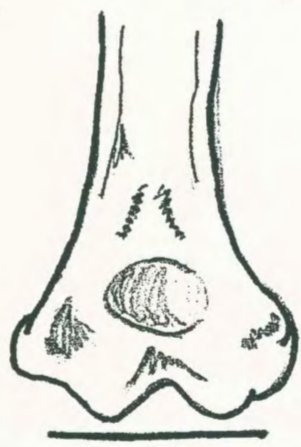

Female

FIG 2: Trochlear Symmetry- in males the trochlea is asymmetrical, in females more symmetrical.

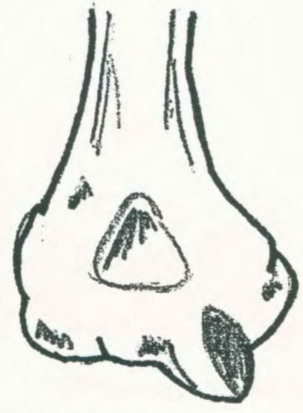

Male

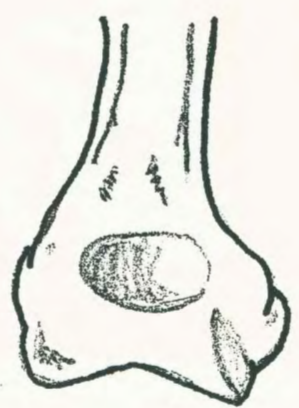

Female

FIG 3: Olecranon Fossa Shape- males exhibit a triangular fossa shape; females exhibit a more oval to round fossa shape. 


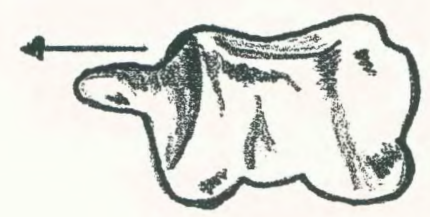

Male

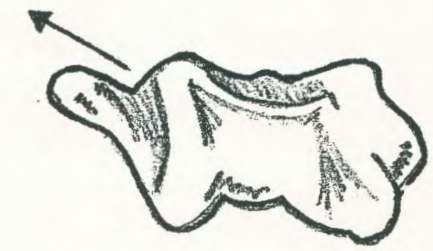

Female

FIG 4: Medial Epicondylar Angle-in males the medial epicondyle is parallel to the table or slightly raised, in females it is raised dramatically.

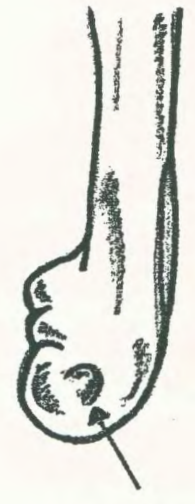

Male

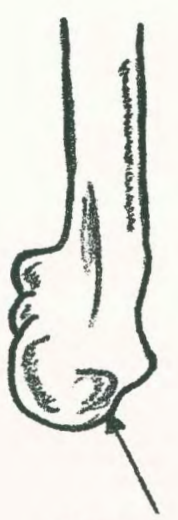

Female

FIG 5: Medial Epicondylar Symmetry- in males, the epicondyle sits more symmetrically within the trochlear profile, in females it sits more towards the posterior, producing anterior asymmetry.
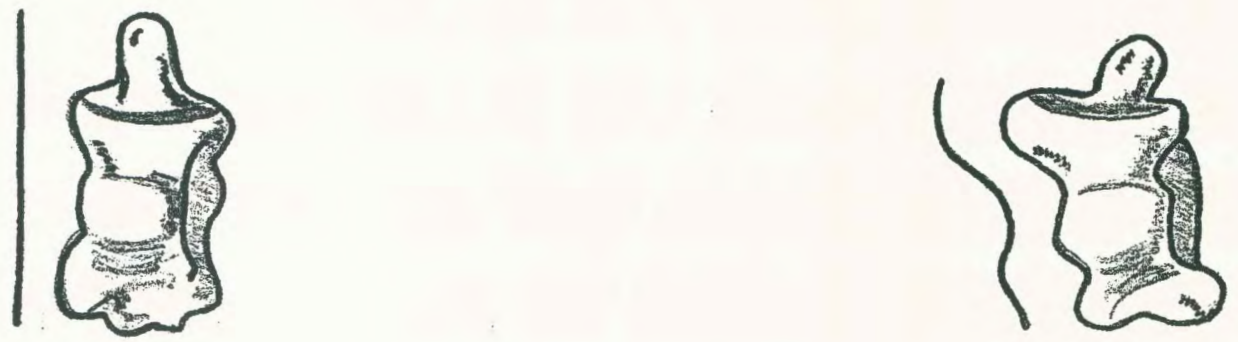

Male

Female

FIG 6: Distal Spool Curvature- the distal spool profile is more perpendicular to the tabletop in males, and exhibits more of a curved profile in females. 


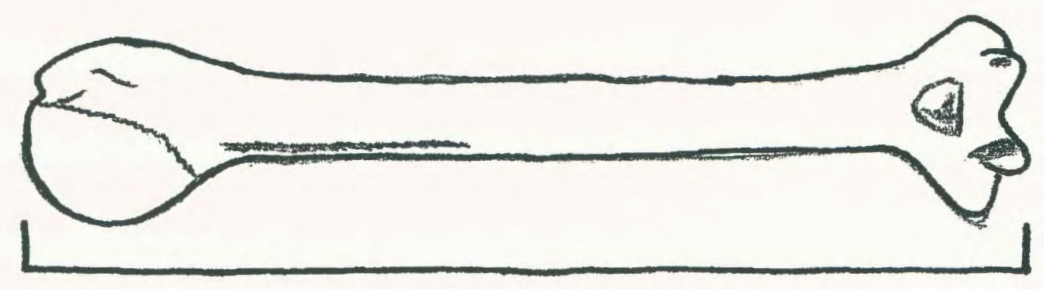

FIG 7: Maximum length of the humerus- the greatest length of the humerus measured with an osteometric board.

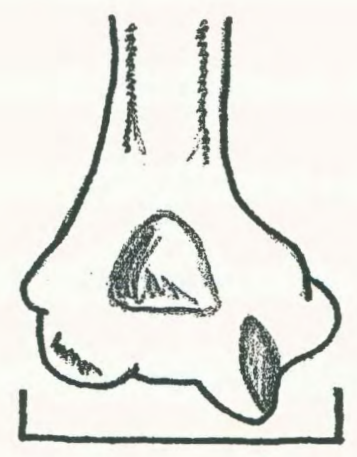

FIG 8: Biepicondylar width of the distal humerus- the maximum distance between the medial and lateral epicondyle, measured with digital metric calipers.

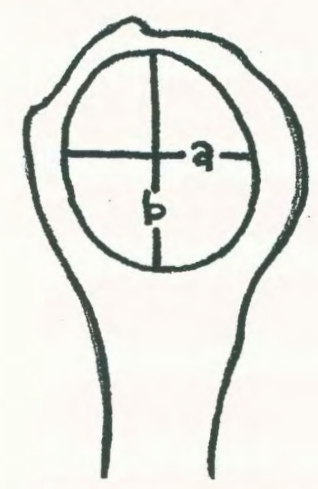

FIG 9: Transverse diameter (a) and vertical diameter (b) of the humeral headtransverse diameter measured perpendicular to the humeral shaft; vertical diameter measured parallel to the long axis of the humeral shaft. 


\section{Chapter III: Results}

I classed each trait as either "male" or "female" (there were no degrees of 'maleness' or 'femaleness'), and I made the final assessment of sex based on the majority of traits assigned. Individuals exhibiting three male traits and three female traits were assigned to "ambiguous". I analyzed a total of 649 individuals. Table 1 figures a division of the total sample by population. I performed chisquare tests on each of the six features to compare sex assignment of each characteristic to known sex, and to determine if any were independent (or dependent on) sex. All characteristics showed a strong association with sex, the relationships between sex and each characteristic were statistically significant, and therefore I concluded that each trait was not independent of sex (see Table 2). I rejected the null hypothesis, and I assessed the statistical significance of each feature in addition to all features combined to establish their accuracy in sex assignment. Of the 649 individuals observed, 487 were correctly identified as male or female based on a consensus of all six features. The accuracy with which each of the six individual traits could predict sex ranged from $60 \%$ to $86 \%$ with all populations included (Table 3). All six traits combined to predict sex correctly $83 \%$ of the time. With all populations and both sexes combined, the olecranon fossa shape was the most accurate individual characteristic ( $77 \%$ accuracy). This accuracy varied among populations, however, from a high of $82 \%$ (American whites) to a low of $60 \%$ (Chinese). Total accuracy of the six combined traits varied among populations, from a high of $94 \%$ (Aleutians, Chinese) to a low of 
$78 \%$ (American blacks). Each characteristic subsequently exhibited varying levels of accuracy between populations (see Table 4). Tables 5 - 11 show individual populations and their respective prediction rates based on each characteristic alone, as well as the combined characteristics documented as "estimated sex".

\section{- American Whites}

Because Rogers' original study (1999) was based solely on a Caucasian sample, characteristics I used predicted the sex of American whites extremely accurately. I was able to correctly predict males $88 \%$ of the time, with the olecranon fossa shape being the most accurate trait (see Table 5). Distal spool curvature was the least accurate predictor at $70 \%$ accuracy. Trochlear constriction was more inconsistent in both males and females, indicating that the variability overlapped considerably between males and females. The other three features (trochlear symmetry, angle of the medial epicondyle, medial epicondylar symmetry) showed variable accuracy. Combined, however, I was able to correctly predict sex at high frequency.

I correctly predicted the sex of white females $82 \%$ of the time. Nearly as accurately, olecranon fossa shape assigned $84 \%$ of the cases correctly. Medial epicondylar symmetry and the distal spool curvature, both predicted sex correctly at $73 \%$. The angle of the medial epicondyle was the least accurate predictor for white females with $71 \%$ accuracy. Overall, I achieved a high rate of accuracywith the combined features, and estimated sex correctly for white females in 82 out of 
100 cases (six ambiguous females were removed from the total number).

\section{- American Blacks}

American black males and females, as predicted, showed less sexual dimorphism and were more difficult to sex using the six diagnostic criteria (see Table 6). More specifically, black females overall showed more male morphology (or what was considered male morphology) than any other group studied. Black females exhibited enormous variation with trochlear constriction and the angle of the medial epicondyle, which predicted sex correctly in only $54 \%$ and $51 \%$ of cases, respectively. This is no better than assignment by "chance". The other features fared better in predicting black females correctly. Olecranon fossa shape was an accurate predictor in females $(85 \%)$, and medial epicondylar symmetry was nearly equally accurate (83\%). Overall, females were correctly classified only $69 \%$ of the time. Black males exhibited a large amount of variation as well, with medial epicondylar symmetry being the worst predictor of sex (46\%). Other features performed better for black males; for example, the angle of the medial epicondyle showed $88 \%$ accuracy. Overall, American black males were correctly sexed in $86 \%$ of the cases, and combined with females exhibited $78 \%$ accuracy.

\section{- Native Americans}

The most accurate predictor of Native American male morphology was the angle of the medial epicondyle, which I classified correctly $83 \%$ of the time (Table 7). Olecranon fossa shape, an accurate predictor with other populations, only correctly assigned $66 \%$ of the Native American males. Distal spool curvature was 
a poor predictor of male morphology, with males exhibiting a curved spool profile (considered a female trait) $57 \%$ of the time. Overall, I correctly classified Native American males $77 \%$ of the time. Females exhibited a different suite of variation. Five of the six features performed quite well with Native American females, with the most accurate (medial epicondylar symmetry) correctly sexing $95 \%$ of the cases. Trochlear constriction, however, was a poor sex predictor in females (58\%). Taken together, all six features correctly assigned $88 \%$ of Native American females. Combined, both sexes of Native Americans were accurately assigned sex $80 \%$ of the time.

\section{- Eskimos}

Eskimos exhibited a range of accuracy among traits. Olecranon fossa shape, as with most other populations, was the best indicator of sex for males and females, with prediction rates of $75 \%$ and $79 \%$, respectively (see Table 8 ).

Trochlear constriction was the least accurate predictor of sex for both males and females. The remainder of the traits fluctuated within a range of accuracy between $57 \%$ and $81 \%$ accuracy for both sexes, and ultimately predicted sex $83 \%$ of the time with males and females combined.

\section{- Aleutians}

The six predictors did a remarkably accurate job predicting sex in the Aleuts (see Table 9). Olecranon fossa shape and the angle of the medial epicondyle predicted sex most accurately in both male and females combined (85\%); symmetry of the medial epicondyle predicted $77 \%$ of males and $83 \%$ of 
females correctly. By contrast, trochlear constriction and trochlear symmetry predicted female sex only $56 \%$ of the time, but accurately predicted male sex $73 \%$ and $86 \%$, respectively. Overall, the six traits accurately sexed both male and female Aleuts $94 \%$ of the time.

\section{- Chinese}

The Chinese sample I analyzed showed an unusual suite of variation compared to other populations I studied. This is figured in Table 10. Males exhibited morphologically female attributes in trochlear constriction, trochlear symmetry and olecranon fossa shape. Despite the poor predictive quality of these three characteristics, the remaining traits predicted sex accurately the majority of the time. The angle of the medial epicondyle predicted males correctly every time, and mis-categorized the lone female as male. The single female in the group exhibited marked female morphology in olecranon fossa shape, medial epicondylar symmetry and distal spool curvature. Overall, I predicted Chinese sex with a $94 \%$ accuracy rate.

\section{- Japanese}

The small Japanese population I analyzed showed, in general, that the prediction properties of the six traits used were accurate (refer to Table 11). Trochlear constriction and the distal spool curvature accurately predicted $100 \%$ of males. Distal spool curvature predicted $100 \%$ of females, while trochlear constriction misclassified the only female. These two traits were characteristically less accurate in other populations analyzed, but resulted in a combined high 
accuracy rate with the Japanese. The other four traits performed well enough to assign sex $80 \%$ of the time with both sexes combined. Japanese medial epicondylar symmetry resulted in misclassifications of the majority of males, while it correctly classified the single female.

\section{- Ambiguous cases}

60 of the 649 individuals I examined had an equal number of female and male traits, and were classified as "ambiguous". Whenever this occurred, I collected four quantitative measurements proven to be reliable for sex determination (France, 1988, Dittrich and Suchey 1986). I did this to ascertain whether quantitative analysis offered better results. I inadvertently did not collect the four quantitative measurements on the "ambiguous" Aleutian cases; therefore I was unable to quantitatively assess this specific group.

Because the qualitative analysis of the sample relied on features with fluctuating statistical significance, I determined which traits performed most poorly, and discarded them in an attempt to more accurately classify sex in the ambiguous cases. I identified two features as having a lower association with sex than the other four characteristics. Trochlear constriction and distal spool curvature did not associate as strongly with sex when analyzing American whites, American blacks, Native Americans, Eskimos, and Aleutians. I removed trochlear constriction from each of the 60 ambiguous cases and re-assigned sex based on the majority. By eliminating this feature, 41 out of 60 ambiguous cases were 
classified correctly. I then removed distal spool curvature to allow for another possible increase in accuracy. With both traits removed, only 35 out of 60 ambiguous cases were correctly sexed. The predictive quality of five features (see Figures 2 through 6) was better than all six features combined when re-analyzing ambiguous cases. Consequently, I reevaluated each ambiguous case to visualize any trends or patterns in their "ambiguousness" and to establish whether quantitative analysis would provide additional accuracy.

\section{- Native American ambiguous cases}

In my analysis of Native American and Eskimo populations, I found seven ambiguous cases. After removing trochlear constriction, six of the seven individuals could be correctly classified. After removing the distal spool curvature, I could only assign the correct sex to two of the seven ambiguous cases. This was a clear indication that removing only trochlear constriction gave more accurate final results. I then used quantitative measurements collected from "ambiguous" Native American and Eskimos and compared them to Dittrick and Suchey's (1986) results from a prehistoric central California Native American sample. All seven of my "ambiguous" determinations would be correctly classified as either male $(n=1)$ or female $(n=6)$ with quantitative methods. Quantitative analysis therefore provided slightly better accuracy than my five significant qualitative features. 


\section{- American black ambiguous cases}

In the American black sample, 21 individuals were ambiguous. When I removed trochlear constriction, 13 of the 21 were accurately assigned. After removing both trochlear constriction and distal spool curvature, 14 out of 21 would have been accurately sexed.

I performed comparative quantitative analysis using the four measurements from all of the ambiguous cases with measurements. Specifically, I used Stewart's (1979) definition of males and females based on the vertical diameter of the humeral head. Stewart's method (published in Bass, 1995) was originally tested on American blacks and American whites from the Terry Collection, and purports to be non-population-specific with these two biological groups. I used Stewart's method first because of its concordance with my technique. Four ambiguous males and nine ambiguous females were correctly assigned sex with Stewart's formula. This figure mirrors the increased accuracy seen when I removed the first visual feature from qualitative analysis.

I then used a second, population-specific quantitative technique (France, cited in Bass, 1995) to assess its predictive value compared to visual techniques. France's method used single variables from different positions on the proximal and distal humerus. It is highly accurate at predicting sex. When bicondylar width, the transverse diameter of the proximal head, and the vertical diameter of the proximal head were combined, the three measurements predicted 19 of 21 ambiguous American blacks. 


\section{- American white ambiguous cases}

There were 17 ambiguous American whites. When I applied the same method of removing the first physical feature, trochlear constriction, 10 were correctly identified as either male or female. When I removed the sixth physical feature, distal spool curvature, 11 were correctly identified as male or female. Based on Stewart's non-population-specific index of the humeral head vertical diameter, only 6 of the 17 ambiguous white individuals were correctly assigned. An equal number of cases (6) were considered "indeterminate" when using Stewart's quantitative technique. France's population-specific formula for sex classification of American whites predicted 13 of 17 ambiguous cases.

\section{- Chinese ambiguous cases}

After removing trochlear constriction, I was able to correctly assign sex to four of the seven Chinese ambiguous cases. When I removed both trochlear constriction and distal spool curvature, only one of the seven ambiguous cases was accurately assessed. Quantitative analysis on the ambiguous Chinese individuals, based on Stewart's vertical humeral head diameter, only correctly sexed the one ambiguous female, and misclassified all the males $(n=6)$. However, this method appears to never have been applied to an Asian population.

France has not published quantitative formulae on Asian populations. Because Northeast Asian populations are considered more genetically similar to Native American populations than to European populations (Relethford, 2001), I applied France's data on Arikara Indians to the ambiguous Chinese. Using 
quantitative methods from three measurements, I was able to correctly assign one Chinese male; the rest were misclassified as female.

\section{- Other ambiguous cases}

Other cases listed as ambiguous, or having an equal number of male and female characteristics included a Puerto Rican individual, a Patagonian, a Korean, a Chilean, and four Alaskan Eskimos. There were no ambiguous cases from the Japanese sample. When I removed trochlear constriction from the suite of features and assigned sex to these "other" individuals, my accuracy increased $100 \%$; in other words, I was able to accurately predict sex for all eight individuals when using the qualitative features \#2 through \#6. Stewart's vertical head diameter index could only correctly predict sex for four of these individuals, indicating the modified qualitative analysis was a better method. France's quantitative formulae for Arikara Indians were again used for the "other" ambiguous individuals based on the most probable genetic similarities. Metric analysis correctly identified five of the eight cases.

In summary, the removal of the first visual characteristic from ambiguous cases allowed me to correctly assign sex to 41 of the 60 ambiguous cases. Quantitative analysis performed well when the biological affiliation of the specimen was known, but performed less accurately than qualitative methods when biological affiliation was unknown and "comparative" population formulae were employed. 


\section{- Individuals misclassified}

Of 649 total cases, I misidentified 102 individuals. Misassignment of sex occurred when I made incorrect determinations in at least four of the six traits visualized. Therefore, the removal of only one trait (trochlear constriction) could never improve the accuracy rate of the method when applied to a misclassification, only serve to "narrow the margin", so to speak, of the misclassification. When both trochlear constriction and distal spool curvature were removed, only 8 of 102 were reclassified correctly. In this specific instance, removing two variables did virtually nothing to improve the accuracy of my visual technique when a case was misclassified.

Of the 102 individuals misidentified, American black females $(n=32)$ were the most common, followed by American white females ( $n=18)$, American black males $(n=15)$ and American white males $(n=10)$. These figures suggest that, in general, females exhibit more male visual morphology than males exhibit female traits. Native Americans were the exception with more males $(n=8)$ misclassified as females than females $(\mathrm{n}=2)$ misclassified as males.

Because I only collected quantitative measurements for a fraction of the total sample observed, I had only 49 with available quantitative measurements to contrast with the results of the visual technique (refer to Table 12). Of these 49 , 26 were correctly designated as male or female with the Stewart vertical humeral head diameter index. Using France's population-specific method, 37 of 49 
misclassified individuals with available measurements were correctly sexed when I applied the appropriate population-specific formulae. 
TABLE 1 - Total sample size by population.

American whites 208

American blacks 234

Native American 54

Alaskan Eskimos 44

Chinese 41

Alaskan Aleutians 40

Japanese 5

Other 23

Total sample size $n=649$ 


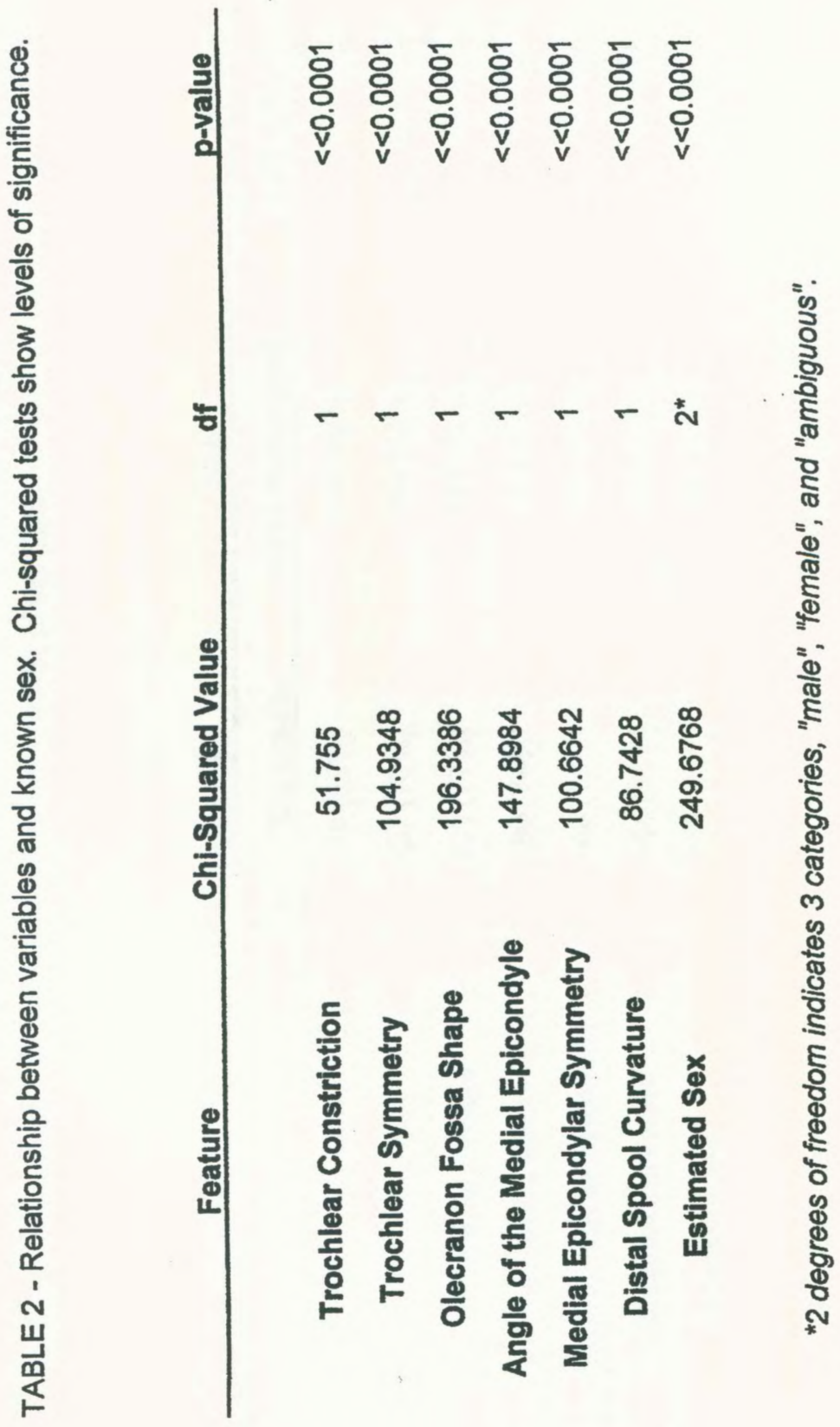




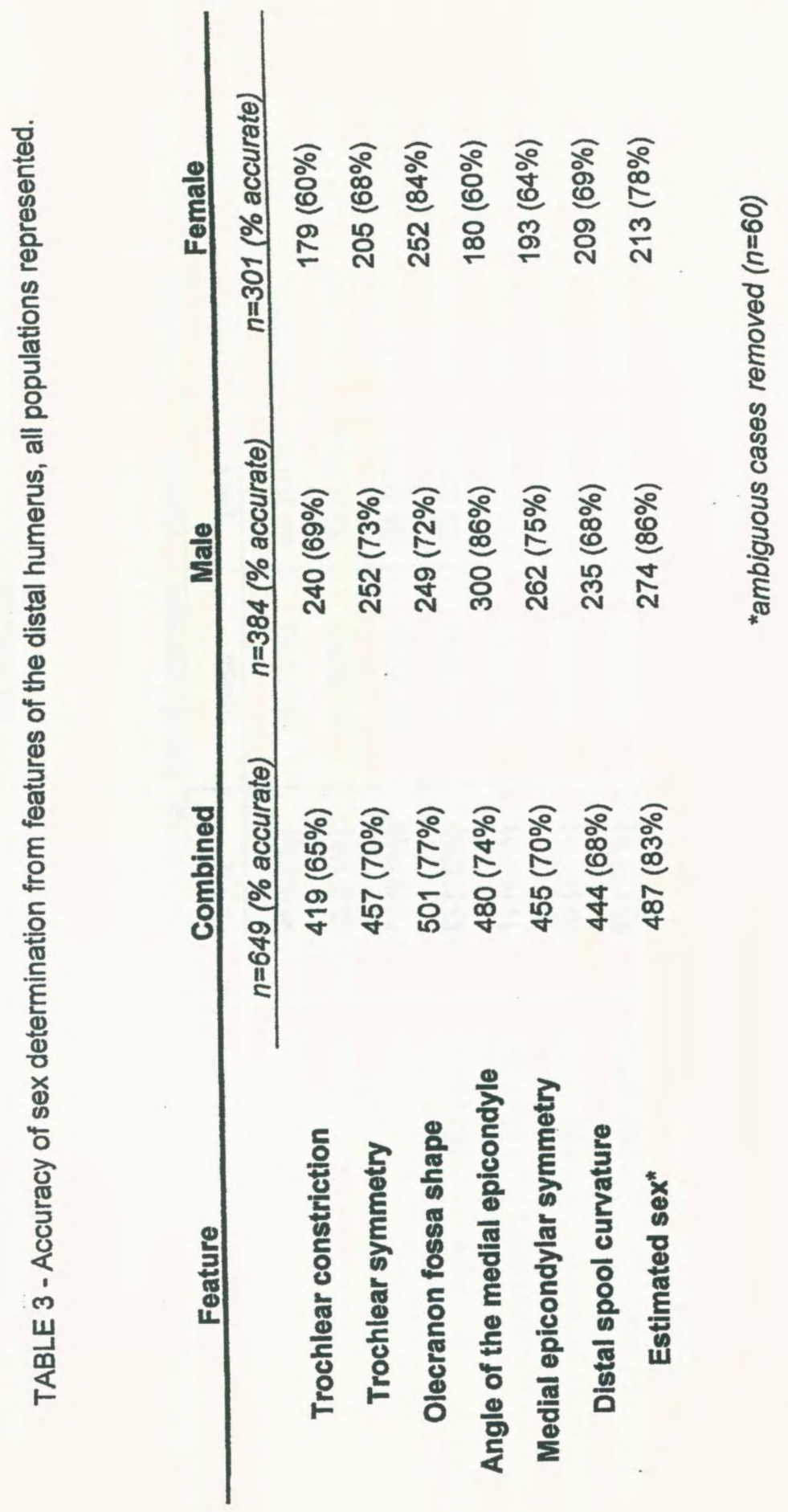




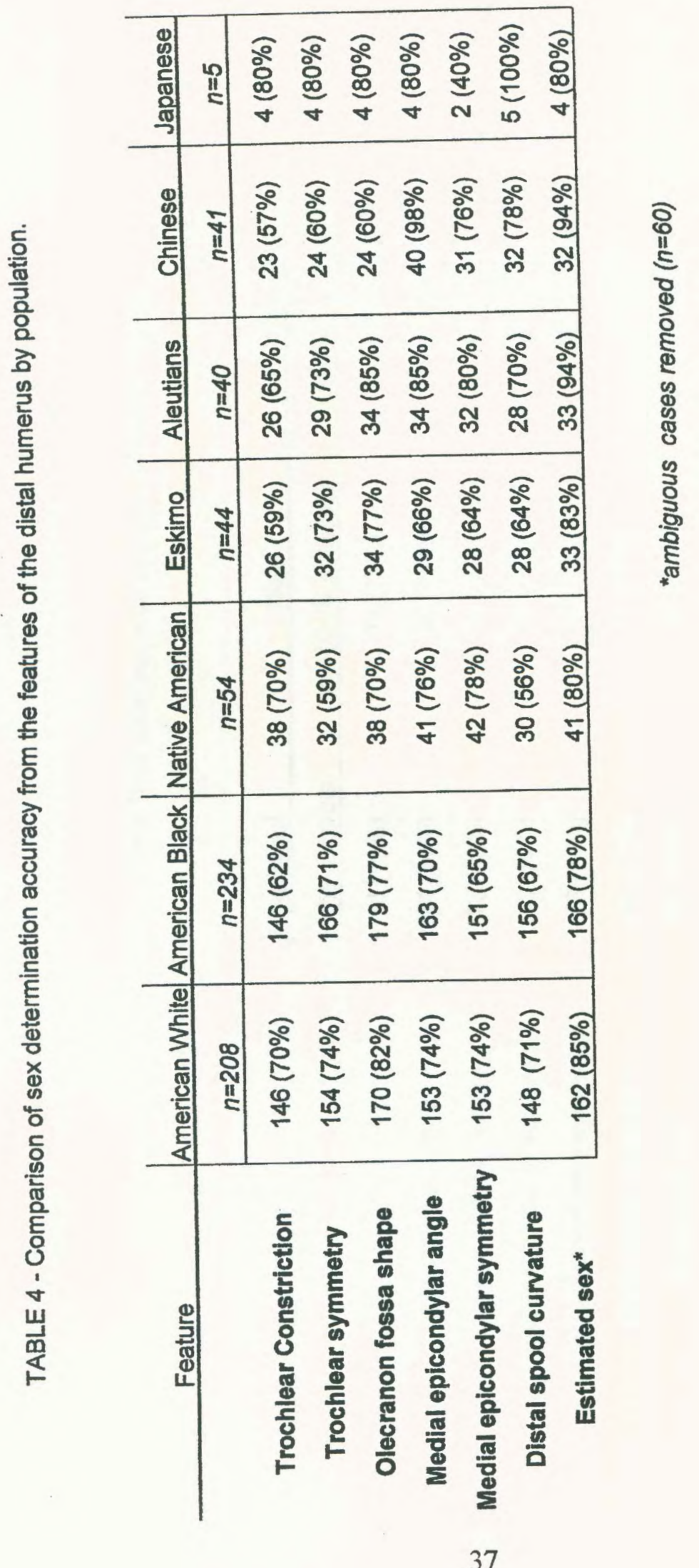




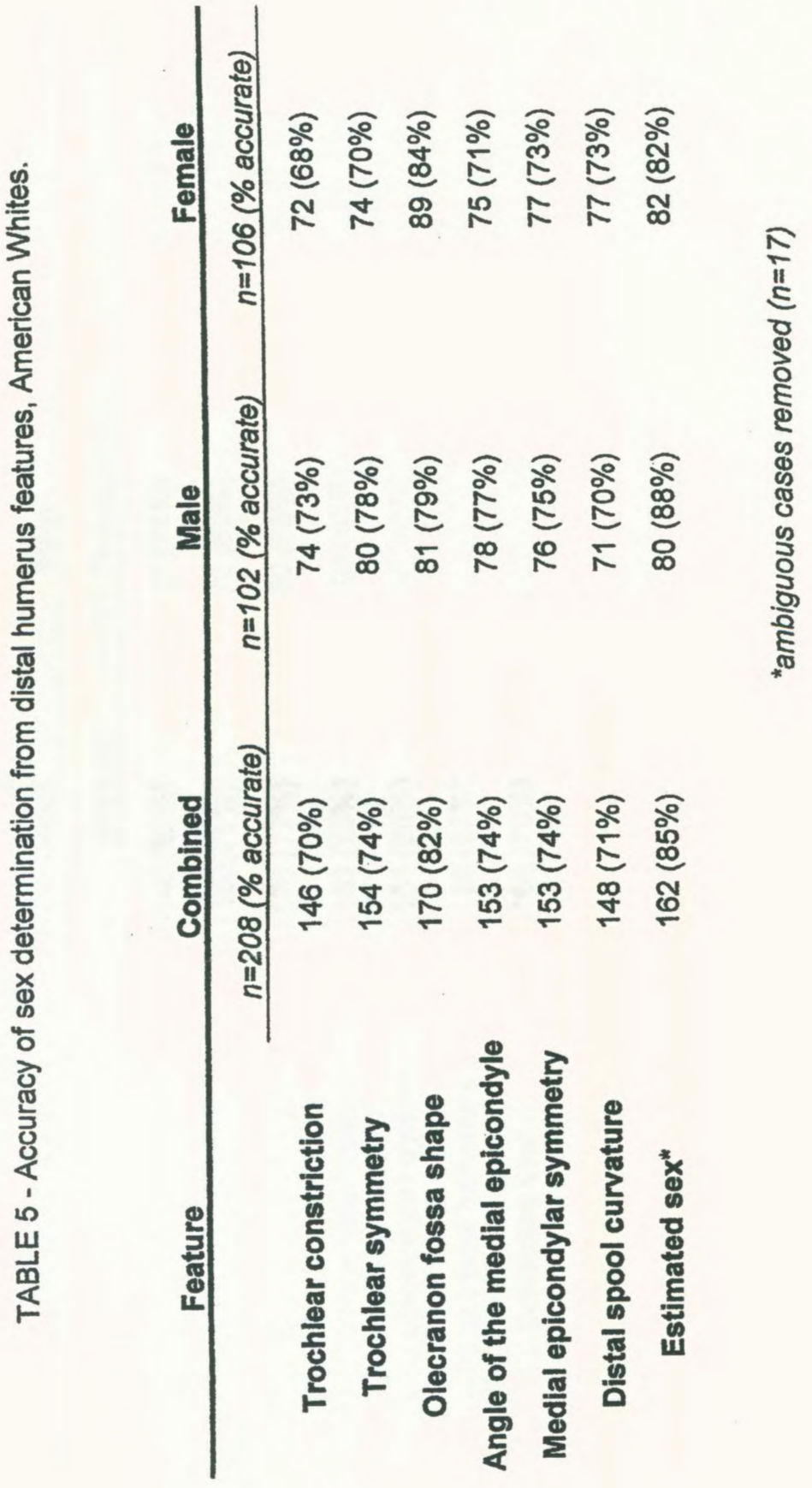




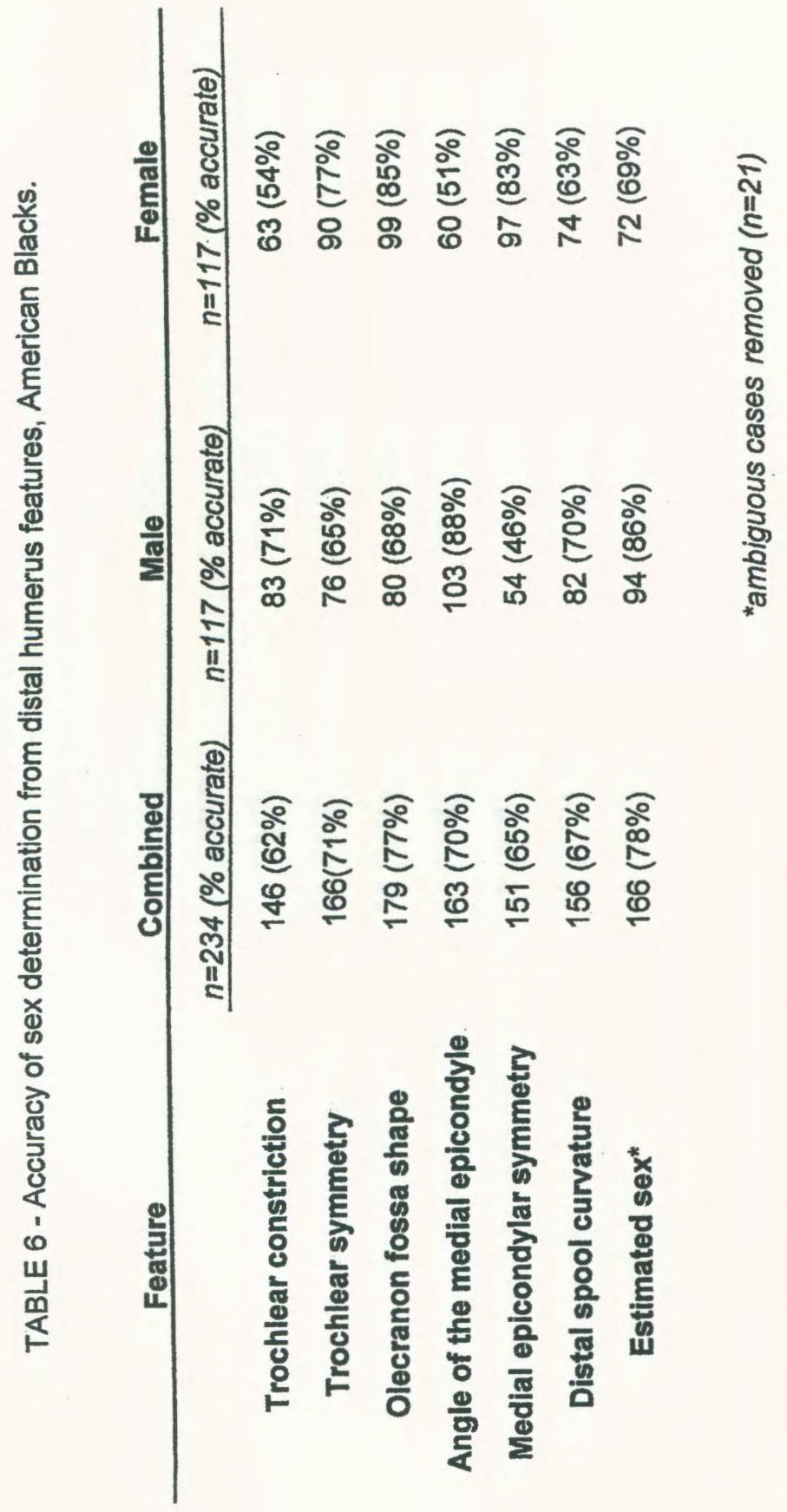




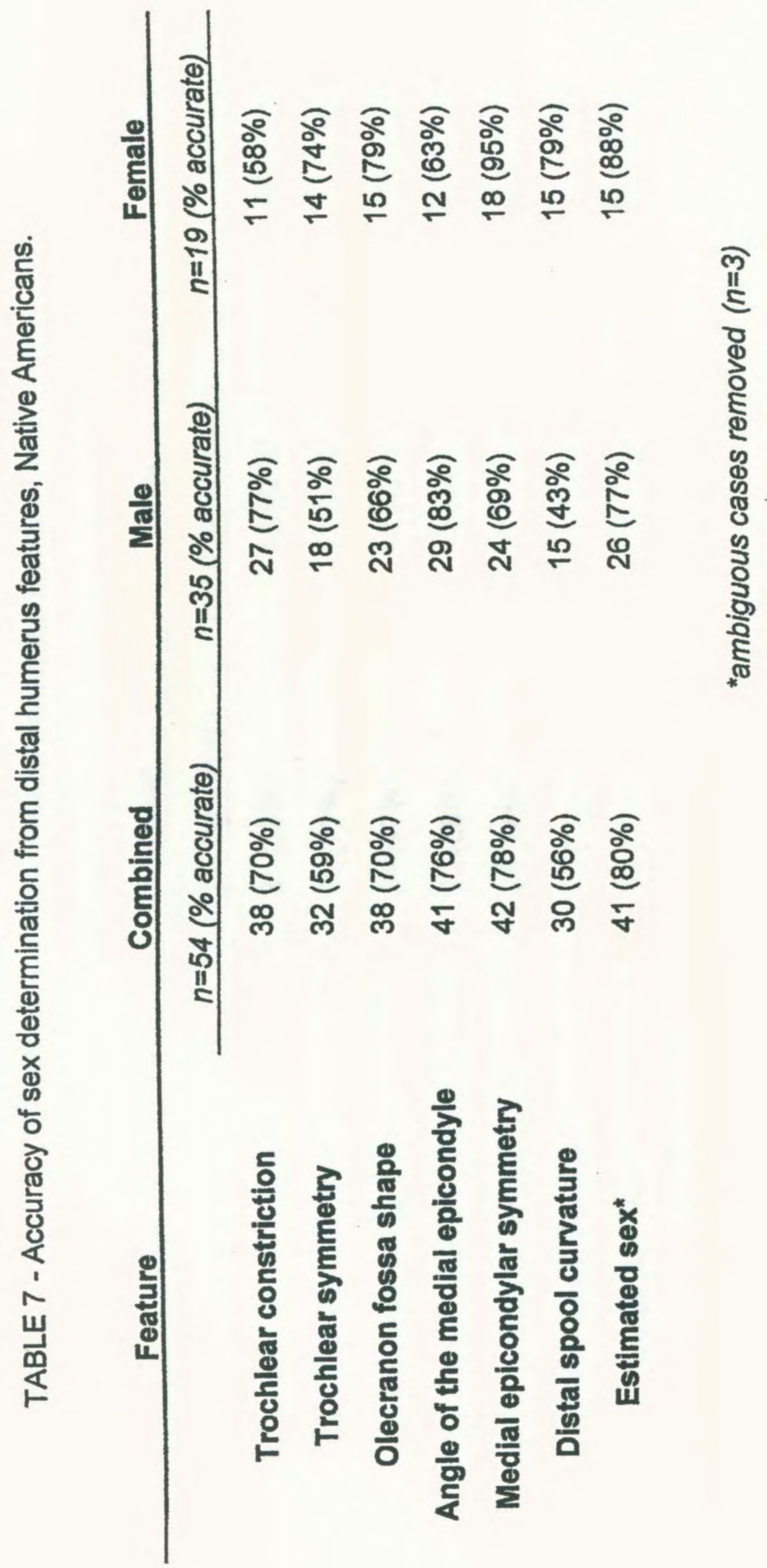




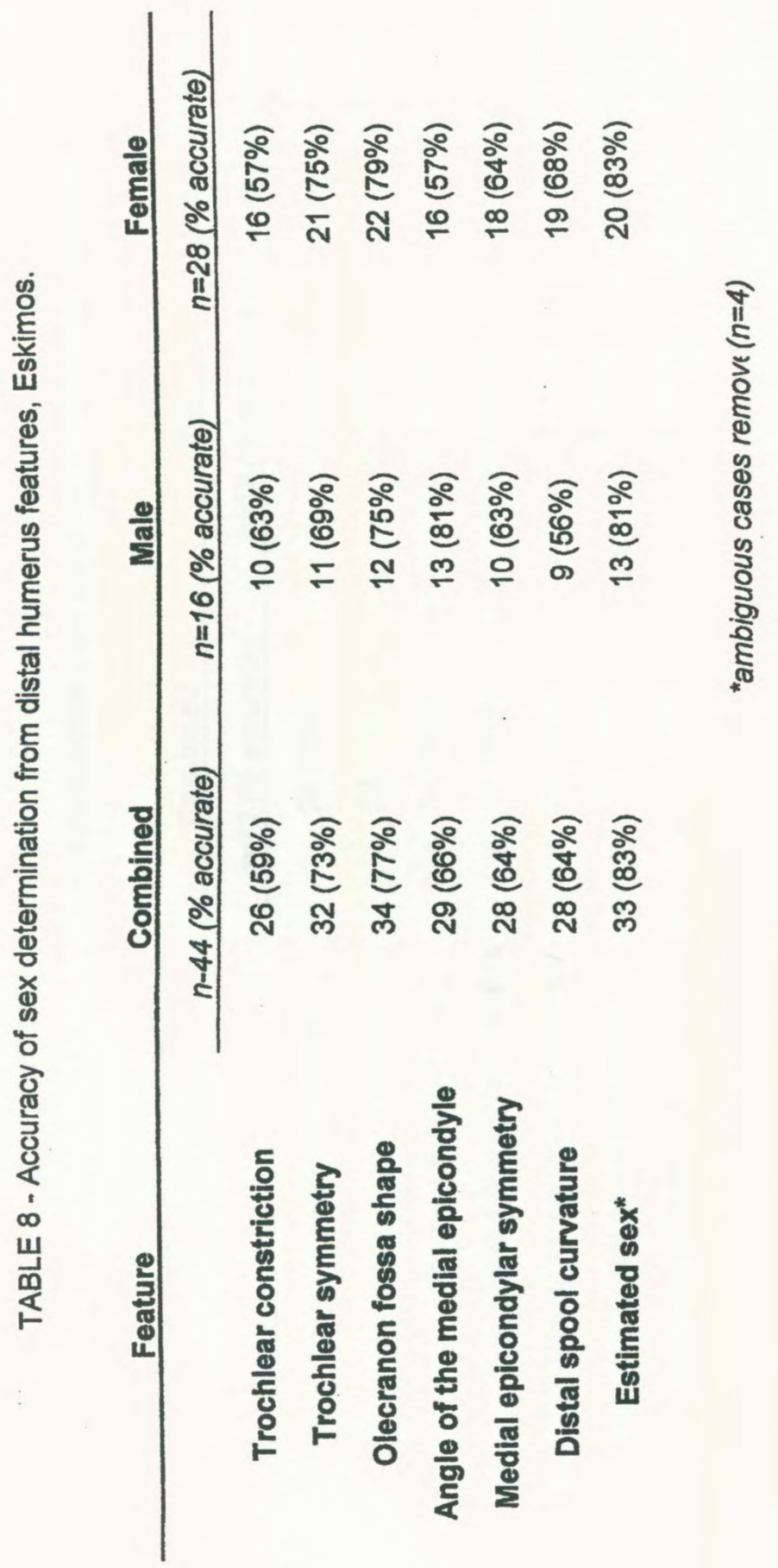




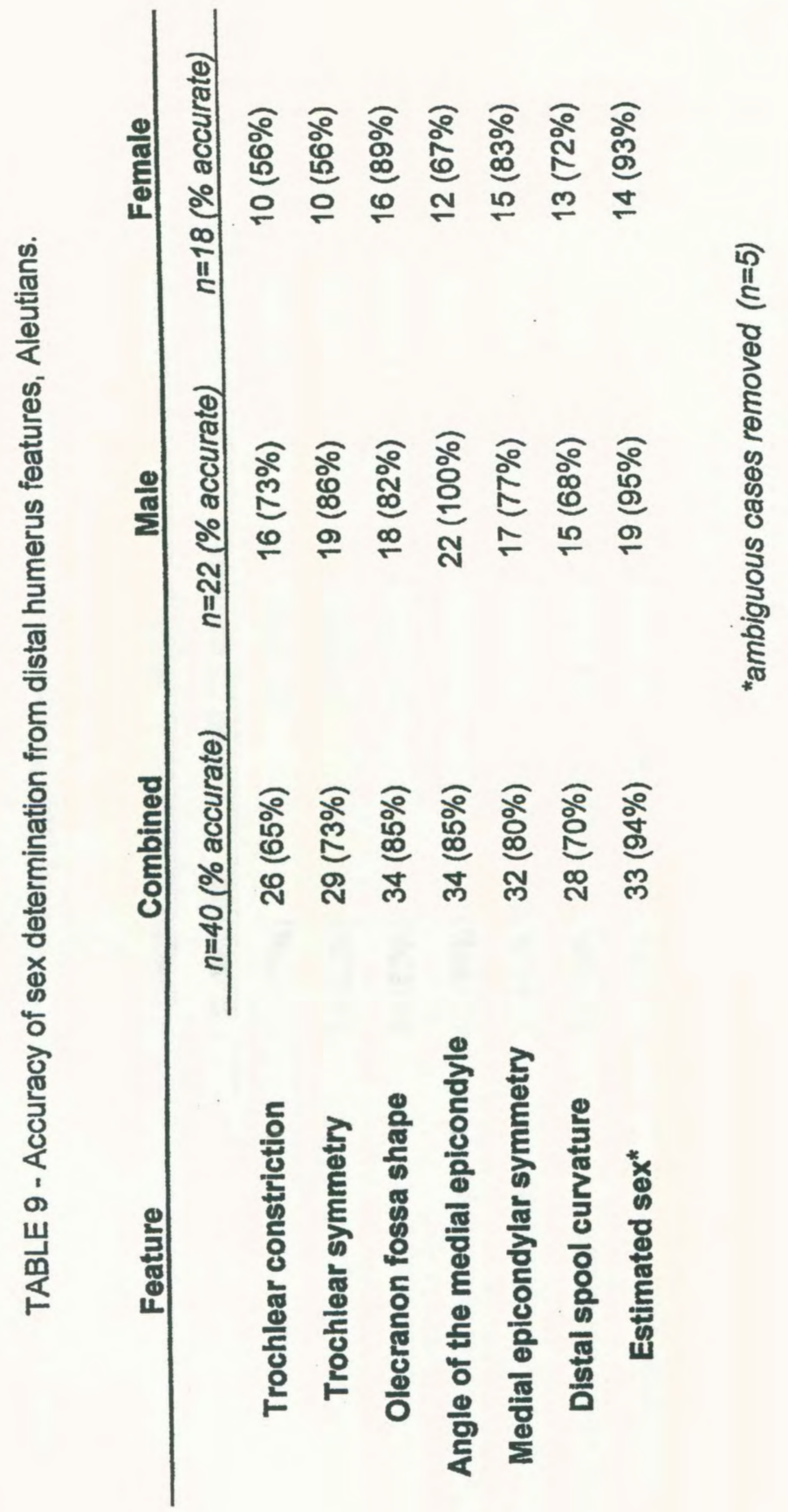




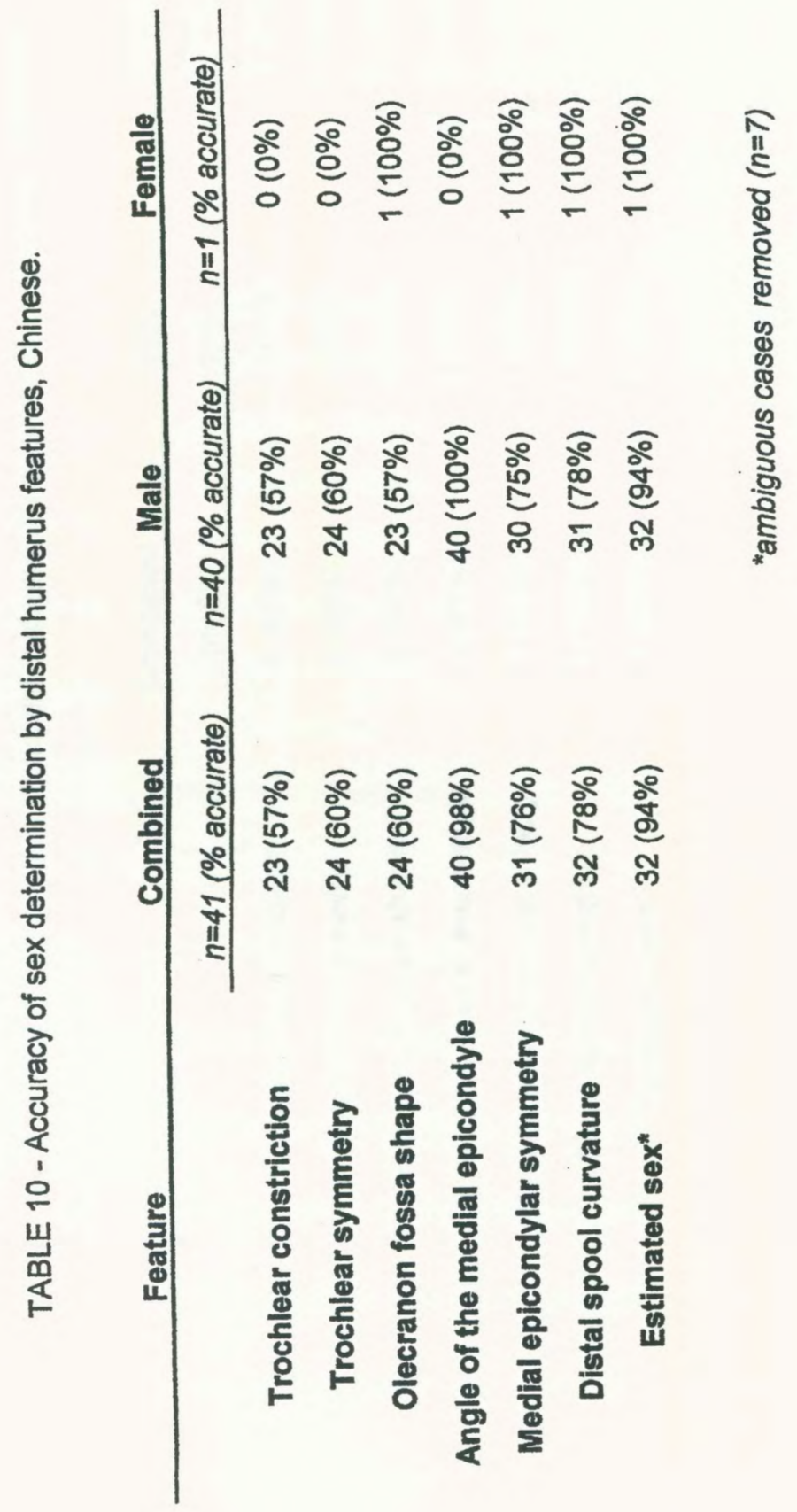




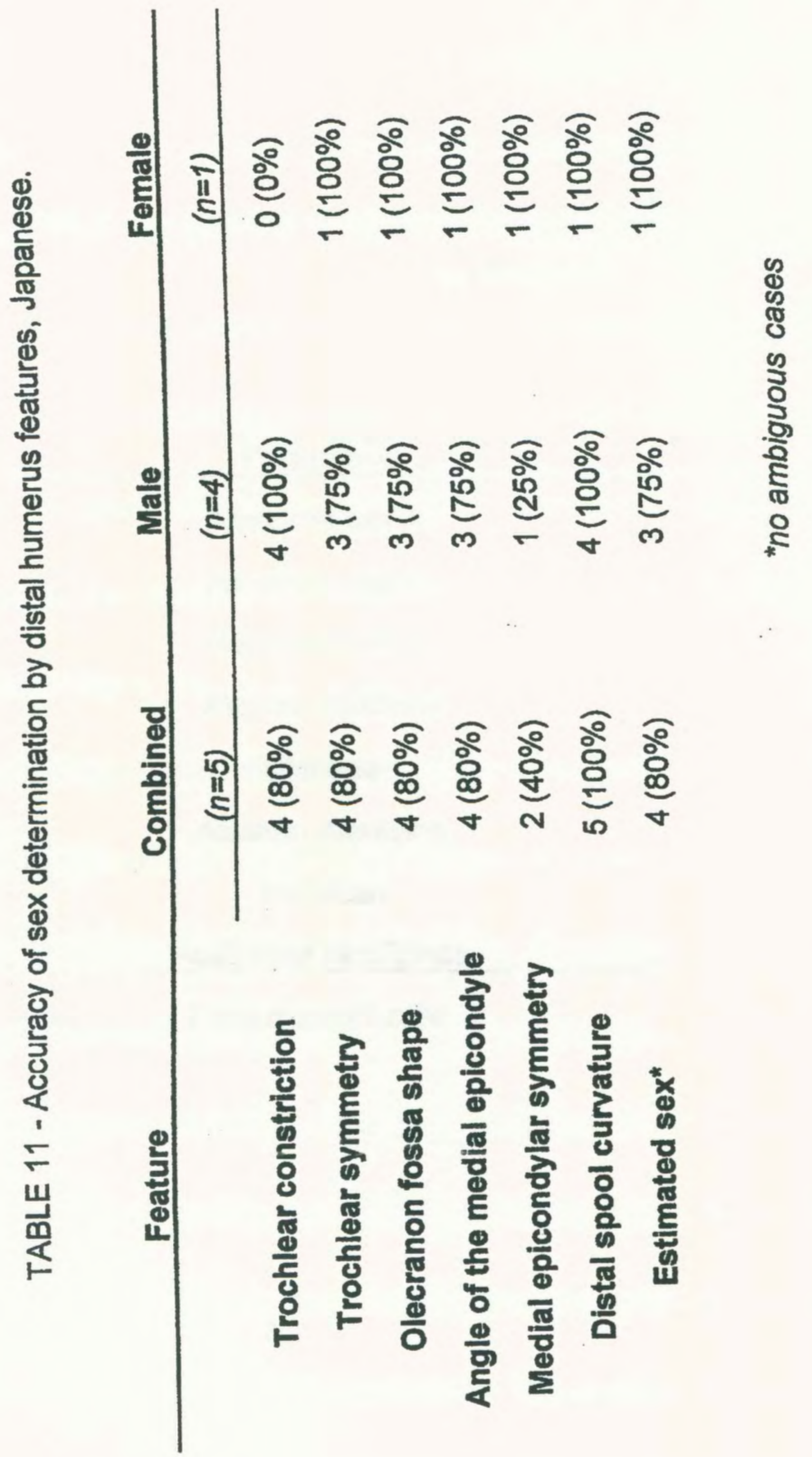


TABLE 12 - Number of misclassified individuals with available measurements, by population.

\begin{tabular}{cc} 
Population & $n$ \\
\hline American whites & 10 \\
American blacks & 16 \\
Native American & 8 \\
Alaskan Eskimos & 6 \\
Chinese & 3 \\
Alaskan Aleutians & 2 \\
Jamaican & 1 \\
Australian Aborigines & 3 \\
Total sample size & $\mathbf{n = 4 9}$
\end{tabular}




\section{Chapter IV: Discussion}

One of the key questions of this thesis was the reliability and efficacy of these methods for different biological groups. Rogers (1999) concluded her study by calling for increased examination of nonwhite populations to determine the applicability of the method across a broad cross-section of humans. White (2000) concurs and urges testing a larger and more wide-ranging sample to further evaluate the qualitative technique. By evaluating numerous biologically diverse populations, I addressed this issue and have exposed the strengths and weaknesses of this technique.

530 total individuals were correctly sexed when I used the modified fivetrait technique. If the remaining ambiguous cases $(n=19)$ are removed, the final predictive quality of this method is $84 \%$. Therefore, the qualitative analysis I performed correctly predicted sex on a statistically significant level $(p<<0.0001)$ when I implemented it with diverse populations. Although my accuracy in assigning sex to individual populations fluctuated between $94 \%$ (Aleutians and Chinese) and $78 \%$ (American blacks), the results indicate a powerfully reliable visual method regardless of population. These results allow me to conclude that my visual method is a dependable sex predictor among diverse population groups.

Another question I presented was whether variations between biological populations were significant enough to note and great enough to make distinctions. I am able to make certain inferences based on the specific populations I studied. Some populations yielded more accurate results than others 
did, which I expected based on prior research. Specifically, American blacks were sexed as I had hypothesized, exhibiting a limited amount of sexual dimorphism, which made a correct determination more difficult. I can also draw certain conclusions based on my observation of individual traits in the American black population. The angle of the medial epicondyle exhibited a poor predictive value for black females (51\%) and a high accuracy rate for black males (88\%). Thus, females exhibited a medial epicondylar angle parallel to the table (considered a male trait) almost as often as they exhibited a raised medial epicondylar angle (considered a female trait). In addition, I found medial epicondylar anterior asymmetry in black males (considered a female trait) more often than I found the trait to be symmetrical (a male characteristic). Overall, because black males exhibited visually female traits and black females exhibited visually male traits, the prediction rate for correctly assigning sex to American blacks was $78 \%$. I observed the same pattern with the Eskimo population; females exhibited the designated male characteristic of a flat medial epicondylar angle quite frequently. To a more limited extent, I obtained the same result from the Chinese population. This begs the question; should I develop separate criteria for the qualitative analysis of American blacks assuming the biological group "black" was known a priori? Should specific criteria be developed for Eskimo and Chinese populations as well? Do these specific populations vary so greatly as to warrant a populationspecific qualitative method? At this point, no pattern appears to exist. These three populations are extremely different from each other in size, and show no 
other similarity to each other in degree of sex determination accuracy with the other traits. Even though American blacks could not be sexed as accurately as others, and even though some females in certain populations exhibited male morphology, it is still possible to determine sex accurately without knowing anything about population affinity.

Different biological populations have varied accuracy when I applied the qualitative method, but only to a certain extent. There was no population that performed under $80 \%$ accuracy except for American blacks. This indicates a strong non-population-specific component of my visual technique. No matter what biological population was set in front of me, I was able to accurately assign sex $83 \%$ of the time with six features and $84 \%$ with the modified five-feature technique. These results are comparable and almost equivalent to Rogers' original research, which was only applied to a relatively small number of European whites. This is a strong argument for the reproducibility, testability, and ultimate accuracy of my modified method in assigning sex to multiple populations, regardless of the physical size of the element. In addition, the creation of two new features (and the subsequent removal of one of the original features) played a role in more accurate determinations. Trochlear constriction (one of Rogers' original features) was removed and the remaining five traits provided increased accuracy when ambiguous cases arose. 


\section{- Revision and discussion of distal humerus features}

Visual assessment has been used successfully to determine sex in a number of bone elements, namely the os coxae and the cranium. Specifically, the pelvis offers sexually dimorphic differences in the angle created by the subpubic concavity and the difference in width of the greater sciatic notch (White, 2000). Both of these observations require the analyst to determine a relative "angle" to the feature. For example, an angle approximate to or less than 90 degrees is considered male, and an angle greater than 90 degrees is considered female in regards the sub pubic region; narrow sciatic notches (a notch that only allows room for two of the analyst's fingers within the notch) indicate males, and broad sciatic notches (a notch that allows for more than two fingers within the notch) indicate females. When I scrutinized the poor predictive quality of trochlear constriction, I considered the possibility of revising the feature based on past (and proven) techniques used in sexing pelvic morphology. Trochlear constriction was the least accurate in sex determination of the six traits used. "Constriction", as defined by both Rogers and me, depicted a "more constricted" or "flexed" medial trochlear aspect for females, and a less constricted or more "gentle" trochlear angle when observed in males. When I reconsidered this description, I discovered the need to further improve the definition; "flexed" and "gentle" constriction were too ambiguous, and may have lent to the ultimate ambiguity of the trait itself. A determination of "constricted approximately 90 degrees or less" may better define female morphology, and a determination of 
"constriction of greater than 90 degrees" may better define male morphology.

Because I didn't consider this "degree" of constriction in this study, further examination of photographs and actual specimens would be needed before revising the trait definition, determining its efficacy, and measuring its possible improvement in sexing accuracy.

Trochlear symmetry is another trait I feel may have been more effective if I had implemented an actual "degree" or "angle" of symmetry. If the humeral element had been placed on grid paper, for example, and the actual angle of the extension visualized with a straightedge, I may have been able to view that particular angle in a more clear and precise manner. In this way, an angle close to 90 degrees (indicating that both the medial and lateral trochlear aspects were quite symmetrical) would be considered female; an angle exhibiting a more acute angle (indicating the extension of the medial trochlea far past its lateral edge) would be considered male. The challenge I find with inserting more rigid and predetermined criteria in the method, however, is the loss of ease and simplicity of the method itself. For example, the above two trait revisions I suggested immediately appear to contradict each other; constriction, or the acute angle of the mid-trochlear region is a female trait, yet with trochlear symmetry, an angle close to 90 degrees is also considered female. By contrast, less constriction (and a wider angle) is a male trait with the first feature, while a more acute angle with trochlear symmetry would indicate male as well. This may be confusing to researchers using the method for the first time. I could determine the distinction between the 
two quite easily, but the uninitiated eye may find a more complex set of criteria daunting. One of the pleasantly surprising elements of my data collection was the fact that I completed my observations on 649 individuals in fewer than two weeks total time. The method I used is quick and quite accurate in its existing form, and the addition of further "criteria" for each trait may defeat the goal of simplicity while offering a relatively small return on the time investment.

The angle of the medial epicondyle was an extremely accurate sex predictor for Rogers (1999). I adopted her definition of the trait, stating that "in males the epicondyle is flat or slightly raised, in females it is distinctly raised" (Rogers, 1999). I predicted male medial epicondylar angles in every population more accurately than female epicondylar angles, which may be a reason to revisit the definition of the characteristic. The worksheet I implemented during data collection had three options for the angle of the medial epicondyle; "flat"; "slightly raised" (both considered male morphology), or "distinctly raised" (a female trait). Removing the "slightly raised" option reduces the criterion to either "flat" or "raised". This may increase the accuracy of sexing female individuals with this trait by categorizing females more explicitly; only females may exhibit any type of raised angle, and only males may exhibit a completely horizontal medial epicondylar angle. This subtle revision of the definition may provide more accuracy; any further study I perform on the existing data will entail returning to the individual data sheets, inspecting all "slightly raised" determinations of the medial epicondyle, then evaluating the actual sex of the element. If females 
exhibited "slightly raised" medial epicondylar angles in numerous cases, I would certainly consider revising the definition to increase the effectiveness of the trait in determining female sex.

The remaining three trait definitions (olecranon fossa shape, medial epicondylar symmetry, and distal spool curvature) were very clear; I could find nothing in the definitions themselves that I felt needed improving. Each provided a clean and easily discernable male or female characteristic. The varying accuracy of each I believe is a result of actual variation in the male and female morphology of each population, and not a limitation of the definitions themselves.

\section{- Sources of bias}

My analysis of diverse populations was by and large a blind study. I made every effort to eliminate any potential biased determinations as I observed each skeletal element. However, two populations (Chinese and Aleutians) housed at the Smithsonian proved challenging to categorize without my prior knowledge of the sex of the individual. I was inadvertently informed while performing my analyses that the Chinese population consisted of only males. I chose to observe this population regardless, based on my need to obtain a sample of diverse population groups. As I analyzed the Chinese group, I continued to assign female characteristics to each feature when appropriate. I concluded that one individual was female. When I returned to the skeletal elements, I discovered that the allmale information given to me was inaccurate, and there was in fact one female (the one I sexed correctly) in the Chinese sample. Despite the inaccurate 
information that all were male, I was not biased in assigning the correct sex to the lone female. Even though I assumed I had classified the individual incorrectly, I continued to visualize all female characteristics as "female", instead of inadvertently being influenced by the assumed male determination. This same observation can be made for the male samples as well; many males actually exhibited female characteristics, and I classified them as such. Ultimately, my classification of the Chinese group was $94 \%$ accurate. This accuracy rate may or may not have been influenced by the prior knowledge of sex, but my vigorous attempt to eliminate analysis bias was consistent throughout the study.

The second population, Aleutians, had the sex marked directly on the humeral shaft. By shielding the shaft and any characters or symbols from sight, I attempted to ignore this information. I determined sex with a $94 \%$ accuracy rate for Aleutians; I cannot establish whether or not the symbols on the shaft were influential to my ultimate determination. Again, I vigorously attempted to control all potential bias throughout the study, and I can only hope that it was successful in this population as well. 


\section{Chapter V: Conclusion}

With this research, I set out to answer certain questions about the applicability of a unique visual method for sex determination from a largely overlooked skeletal element, the distal humerus. These questions included:

- What was the reliability of this method for different biological populations?

- Was variation between biological populations significant enough to note and great enough to make distinctions?

- Where does the method break down or misassign sex, and is this misassignment population-specific?

- Are there alternative features that may increase the sexing accuracy? And finally,

- If skeletal elements cannot be assigned to a population, how can accurate results be obtained from applying population-specific quantitative techniques? Can a non-population-specific visual method provide the same or better accuracy as metric techniques?

I determined that the efficacy and reliability of this method was strong when I applied it to several diverse populations. Every group I studied varied considerably in size dimensions, but exhibited common morphological features of the distal humerus. Each biological group had its own suite of variation, but the ultimate outcome of sex determination was quite accurate for all populations. This 
confirms that visual techniques continue to provide accurate results regardless of biological affiliation.

I considered specific qualitative criteria for each population based on the subtle contrast between biological groups. However, each population varied so little when compared to each other; I concluded a population-specific qualitative method would only diminish the simplicity of the technique, and was not warranted. The method was accurate with different biological populations. This strong nonpopulation specific component allows the method to be used when the biological affiliation of the specimen is unknown.

The method broke down and misclassified more females than males. This shows that females exhibit more male morphology than males exhibit female characteristics. More American black females were misclassified than any other population I studied, indicating exactly what I had predicted with decreased sexual dimorphism in black populations. A revision of two original trait definitions may increase the accuracy of sex determination, and provide additional precision in future studies. By and large, the method's shortfalls were insignificant compared to its positive implications as a legitimate qualitative technique.

Finally, I felt the unconditional acceptance of most quantitative methods required further scrutiny and review. Almost always, these methods are based on the gross size of the element. Comparison of different biological populations reveals the wide range of human size variation; when analysis is restricted to size alone, researchers may never accurately distinguish between a large female and a 
small male. In addition, metric analysis is never $100 \%$ accurate, even when applying the correct formulae to the correct population. One of my goals in this research was to reintroduce the accuracy and applicability of visual techniques largely disregarded by those who implement measurements. If metric features hold more analytical weight than non-metric features when determining sex, researchers are limited to the size of the element without considering the distinctive form of the element; they limit themselves to the data and regression formulae only available for a limited number of population groups; and they limit themselves to only those bone elements that are intact and in pristine condition. Visual assessment of the distal humerus provides an accurate, reliable sex determination method without these three parameters. The six distinct physical characteristics of the distal humeral element I analyzed were easy to visualize, and the method was quicker than collecting measurements for quantitative analysis. When compared to metric techniques, the visual method I used was analogous in accuracy to quantitative measurements, and never required a correct biological or "racial" assignment to be successful.

The distal humerus was proven (Rogers, 1999) to exhibit marked sexual dimorphism in European Caucasians. I have effectively demonstrated in this study that it exhibits marked sexual dimorphism in several other populations as well. This visual method can be used with confidence alone or in conjunction with other skeletal elements to accurately determine sex of unidentified remains. The implications of this method as a field technique are wide-ranging. Physical 
characteristics of the distal humerus may now be employed more frequently and with confidence when more conventional diagnostic skeletal elements are absent. In addition, when faced with remains from several different biological populations, this visual method can be used as a powerful tool in sex determination. The biological affinity of the specimen analyzed does not play a role in this technique, eliminating any kind of potential misclassification based on size variations between populations. This should encourage analysts to use morphological characteristics from the distal humerus in cases of archaeological interests such as historical burials, in contemporary forensic settings for unidentified scattered remains, and in mass disasters such as plane crashes and catastrophic events where remains are fragmented and/or co-mingled. The distal humerus and its unique physical features provide an exciting alternative or supplementary method to previously used quantitative methods, and I have demonstrated its effectiveness as a visual technique, as well as its applicability to diverse population groups and its ultimate accuracy in sex determination. 


\section{$\underline{\text { References }}$}

Bass, W. (1995) Human Osteology: A Laboratory and Field Manual. $4^{\text {th }}$ Ed. Columbia, MO: Missouri Archaeological Society.

Burns, K. R. (1999) Forensic Anthropology Training Manual. Prentice Hall Inc., New Jersey.

Dittrick, J. and Suchey, J.M. (1986) Sex Determination of Prehistoric Central California Skeletal Remains Using Discriminant Analysis of the Femur and Humerus. Am J Phys Anthropol, 70:3-9.

France, D.L. (1988) Osteometry and Muscle Origin and Insertion in Sex Determination. Am J Phys Anthropol, 76:515-526.

Grabiner M.D. (1989) The elbow and radioulnar joints. In: Rasch PJ, editor. Kinesiology and applied anatomy. London: Lea and Febiger, 1989. As cited in Rogers, TL. A Visual Method of Determining the Sex of Skeletal Remains Using the Distal Humerus. J Forensic Sci, 44(1): 57-60.

Holman, D.J. and Bennett, K.A. (1991) Determination of Sex From Arm Bone Measurements. Am J Phys Anthropol, 84:421-426.

Iscan M.Y., Loth S.R., King C.A, Shihai D. and Yoshino M. (1998) Sexual Dimorphism in the Humerus: A Comparative Analysis of Chinese, Japanese and Thais. Forensic Sci Int, 30(1-2): 17-29.

Jantz, R.L., Hunt D.R. and Meadows L. (1994) Maximum Length of the Tibia: How Did Trotter Measure It? Am J Phys Anthropol, 93: 525-528.

King, C.A., Isca, M.Y. and Loth, S.R. (1998) Metric and Comparative Analysis of Sexual Dimorphism in the Thai Femur. J Forensic Sci, 43(5): 954-958.

Mall G., Hubig M., Buttner A., Kuznik J., Penning R. and Graw M. (2001) Sex Determination and Estimation of Stature from the Long Bones of the Arm. Forensic Sci Int, 117 (102): 23-30.

Relethford, J.H. (2001) Genetics and the Search for Modern Human Origins. John Wiley \& Sons, Inc., New York, NY.

Richman E.A., Michel M.E., Schulter-Ellis F.P. and Corruccini R.S. (1979) Determination of Sex by Discriminant Function Analysis of Postcranial Skeletal Measurements. J Forensic Sci, 24:159-63. 
Rogers, T.L. (1999) A Visual Method of Determining the Sex of Skeletal Remains Using the Distal Humerus. J Forensic Sci, 44(1): 57-60.

Saunders S.R. and Hoppa R.D. (1997) Sex Allocation from Long Bone Measurements using Logistic Regression. Can Soc Forens Sci J 30(2): 49-60.

Shipman P., Walker A., Bichell, D. (1985) The Human Skeleton. Harvard University Press.

Steele, D.G. and Bramblett, C.A. (1988) The Anatomy and Biology of the Human Skeleton. Texas A\&M University Press.

Stewart, T.D. (1954) Sex Determination of the Skeleton by Guess and by Measurement. Am J Phys Anthropol, 12:385-92.

Stewart, T.D. (1979) Essentials of Forensic Anthropology. Springfield, Illinois: Thomas.

Steyn M. and Iscan M.Y. (1999) Osteometric Variation in the Humerus: Sexual Dimorphism in South Africans. Forensic Sci Int, 106(2): 77-85.

Tanaka H., Lestrel P.E., Uetake T, Kato S. and Ohtsuki F. (2000) Sex Differences in Proximal Humeral Outline Shape: Elliptical Fourier Functions. J Forensic Sci 45(2): 292-302.

VanDongen, R. (1963) The Shoulder Girdle and Humerus of the Australian Aborigine. Am J Phys Anthropol, 21(4): 469-487.

White, T.D. (2000) Human Osteology ( $2^{\text {nd }}$ Edition). Academic Press, San Diego, CA. 


\section{General Information:}

$\log \#$

Feature \#1: Trochlear constriction

Less constricted medial portion $\mathbf{M}$

More constricted, spool-shaped

F

Weak constriction $\mathbf{M}$

Dramatic flexion F

Ambiguous

Feature \#2: Trochlear symmetry

Medial trochlea extends distally $\mathbf{M}$

Lop-sided $\mathbf{M}$

Medial, lateral edges almost equal $\quad$ F

Less lop-sided $\mathbf{F}$

Ambiguous

Feature \#3: Olecranon fossa shape

Triangular $\mathbf{M}$

Oval F

Oval with proximal extension $\mathbf{F}$

Ambiguous

Feature \#4: Angle of the medial epicondyle

Parallel to the tabletop M Raised angle F

Slightly raised $\quad \mathbf{M}$

Ambiguous

Feature \#5: Medial epicondylar symmetry

Positioned centrally within trochlear profile $\mathbf{M}$

Fingers rest on both shelves $\quad \mathbf{M}$

Anterior symmetry $\quad$ F

Ambiguous

Feature \#6: Distal spool curvature

Perpendicular to tabletop $\mathbf{M}$ Curved profile $\mathbf{F}$

Ambiguous

Determination: M F

Other distinguishing characteristics:

Length $=$

Bicondylar width $=$

Sex:

Population:
Transverse Diameter $=$

Vertical Diameter= 


\section{APPENDIX B: COLLECTION BACKGROUND, LAB PROTOCOL, DOCUMENTATION AND OBSERVATION TECHNIQUES}

\section{- The Hamann-Todd Human Skeletal Collection, Cleveland Museum of Natural History, Cleveland, Ohio}

Extensive records kept on the cadavers from the Hamann-Todd Human Skeletal Collection include death certificates, clinical histories, and anatomical reports of past injuries or pathology if available, photographs, some hair samples, and in numerous cases plaster-of-Paris "death masks". Being considered "the unclaimed dead of Cleveland", low socio-economic status and substantial population growth during the time period of active cadaver procurement explains the high representation of blacks in the cadaver population. Those whose bodies were identified and donated largely were done so in the effort to procure funds from the donation.

Causes of death listed for each individual indicate the pathological profile of the era; the most common causes of death were listed as respiratory tuberculosis, heart disease, pneumonia, alcoholism, apoplexy, and nephritis. The age of the individual was recorded when the bodies were received into the museum, and then determined again after maceration and skeletal assessment. Maceration of the cadavers was accomplished by complete dissection and disarticulation by knife and subsequent scraping of additional connective tissue and air-drying. No artificial drying techniques or chemicals were utilized in order 
to maintain a certain amount of fats for long-term preservation (Cobb, 1932). I accessed the Hamann-Todd collection through the assistance of Dr. Bruce Latimer, CMNH's Director and Mr. Lyman Jellema, the collections manager at the museum.

- The Terry Human Skeletal Collection, National Museum of Natural History, (Smithsonian), Washington, D.C.

Dr. Robert J. Terry, head of the Anatomy Department at Washington University Medical School in St. Louis, Missouri began to collect human skeletons from cadavers used in the Medical School's anatomy classes in an effort to successfully procure viable reference material for teaching purposes (Hunt, pers.comm.). An established protocol for the collecting, cataloging, maceration and storage of the collection was developed documenting morgue records with the name, sex, age and "ethnic identity", cause of death and date of death of each individual. Maceration was completed by stripping the bone of as much tissue as possible without damage, soaking the skeleton in hot water for 72 hours, brushing the bone with a coarse-bristled brush, and then finally drying the bone.

Degreasing of the bone was accomplished by exposure to benzene vapors to remove fats. The collection was transferred to the National Museum of Natural History, Department of Anthropology in 1967 after a change of focus in St. Louis Medical Schools from skeletal morphology to research in brain morphology and function.

The Terry Collection (unlike the Hamann-Todd Collection) housed not 
only individuals of poor socio-economic backgrounds, but also a large number of white females who, during the 1920's and 1930's, found it quite in vogue to donate one's body to scientific endeavors. This supplemented an area of the collection that may have very well gone lacking in sample size if not for the social implications and cultural beliefs of the era.

\section{- Lab Protocol, Cleveland Museum of Natural History}

Upon my arrival, Mr. Jellema pulled $300 \log$ numbers and generated a list including the criteria of age, race, and birthdate. The four sub lists generated produced a sample of 75 black females, 75 white females, 75 black males and 75 white males all chosen at random and representing a comprehensive sample of biological affiliation. I also asked and received the humeral elements from only those individuals of a specific age group, namely $25-45$ years old. This would theoretically eliminate most age-related pathology common in older individuals. Lyman and his volunteer, Ms. Lillian Rubin then retrieved the individuals based on the numbers chosen at random by the database. Those assisting me took great care in carrying out my specific requirements. I was not allowed near the storage rooms while they were retrieving the humeral elements from each box, and they took great pains to make sure the humeri were placed randomly on a transportation tray, so as not to group one sex or one race together. Only after making quite sure they had adhered to my need to have a fully blind test sample did they allow me to enter the room in which I would be collecting data. Each element was placed together on a foam-lined rolling tray for convenience of transportation, and 
transferred from the storage area to the analytical area. This analytical room was expansive in its dimensions and allowed me to line a number of bones along a long counter top surface in order to view them more readily. Once the bones were placed on the counter top, I set up a surface for observing the distal portion of each bone. This consisted of a piece of foam approximately 2 -feet by 2 -feet covered with a section of black tight-weave velvet. The lighting within the laboratory was adequate, so I did not require additional illumination until photography warranted it. Because my observations of the distal element required me to stand during most of the analysis, but crouch to align my eyes with the countertop level in other portions of the analysis, standing became the preferred method of observation and was continued throughout the week.

Furthermore, I was not allowed to enter into the storage location until the last day of my data collection. Only after the accumulation of all needed information was I allowed to enter the storage rooms to take photographs and observe how the individuals were curated.

\section{- Lab Protocol, Smithsonian Museum}

Numerous components of my original analysis present at the Cleveland Museum of Natural History were absent in the analysis of humeri from the Smithsonian. The Smithsonian's numerous collections of curated remains comprise almost 30,000 individuals of diverse biological affiliations, affording me an opportunity to collect information essential to testing the hypothesis of a nonpopulation-specific technique. However, many of the different collections were 
curated in groups of bone elements, and not as individuals with specific information. For example, a box of Peruvian humeri would have provided me with at least 40 individuals to collect information from, but none of the elements had been accurately sexed, and could not be matched back to their appropriate crania or pelves. Therefore, large portions of different collections had to be ignored based on the fact that they had never been definitively sexed, and the individual bone elements had never been entered into a database for reference. In addition, when I inquired about different Asian populations possibly curated at the museum, the Chinese population was inadvertently revealed to me as all male, which would eliminate any blind study conducted upon the Chinese humeral elements. I decided to look at the entire sample regardless of the knowledge that they were all male to attempt to discern any dissimilarities in Asian males as opposed to white or black males. While conducting the analysis of Asian males, I "misclassified" two individuals as females. When returning the humeri to their appropriate boxes, I discovered that two of the Chinese skeletons had been misidentified as male by previous researchers; both were markedly female in pelvic morphology and exhibited written female designations on all the other bone elements, including the humerus. In raising this issue with Dr. Hunt, he concluded that they had been mis-categorized in the database as male, and were in fact female. This was one distinct instance in which the visual technique I was applying to humeri correctly assigned sex, and caught a mistake in the database sample. 
An analytical space was provided in "The South Dakota Room" of the Physical Anthropology Department, and an area consisting of an approximately two-feet by two-feet section of foam with an overlay of black tight-weave velvet was prepared. I identified individual specimens either as part of the Terry Collection or as a part of another collection, located in another annex of the museum. I removed left humeri from individual boxes and placed them on a foam-lined transportation cart. When I had acquired an adequate sample, I transported the cart back to the analytical space for observation of each bone. The lighting within the room was adequate, so I did not require additional illumination until photography warranted it.

Additionally, individuals that comprised the Terry Collection all exhibited labeling documenting the age, sex and race of each on the external surface of the container in which they were stored. This was of great concern to me, due to the fact that I considered a blind study of the bones to be the most accurate and unbiased indicator of the technique's efficacy. I personally went to each specimen and removed the left humerus from storage; this was an additional concern of mine, knowing that $\mathrm{I}$ had seen the accurate sex and race of the individual before making any qualitative determination from the element. However, the sheer number of specimens I looked at one time was sufficient to prevent any potential bias due to the inadvertent memory of the correct sex for an individual. It was not unusual for me to take a foam-lined transportation cart to the Terry Collection area, begin in one location, and work through a section of containers quite quickly, 
without looking at the sex and race determination on the container's exterior.

I by in large disregarded the proximal head and shaft during the initial examination of the distal humeral portion, and in the case of non-Terry Collection skeletal elements, I used a small piece of black cloth to cover any documentation listed on the bone itself. This obscuring technique eliminated most unintentional bias when observing the element. Notations were made close to the proximal head of the humerus, so obscuring the sex determination was quite simple in most cases. When the inscription was exhibited on the distal shaft, however, the difficulty in keeping that particular portion of the element covered increased, based on the fact that I had to observe the distal end to make my determination. To compensate for this potential self-bias, I recorded the known sex of each individual in two different ways. The known sex of those humeri included in an ambiguous and essentially blind collection, with no distinguishing markings or indicators of sex on the bone itself, were recorded in red ink in the analytical notebook. The known sex of those humeri included in a potentially bias sample, with inscriptions indicating the individual's sex located somewhere on the element, were recorded in black ink in the analytical notebook. I did this in an effort to control and perhaps measure the bias that I inflicted on my own study. Those individuals whose actual sex is denoted in red are the truly unbiased, blind representations of the study. Those documented in black may have fallen unintentionally within a biased framework, based on whether or not I inadvertently exposed the inscription on the bone, thus exposing myself to classify the bone a 
certain way, not based on the physical characteristics of the study but based on what I had visualized on the humeral shaft.

\section{- Documentation}

Originally, I developed a worksheet much like the one shown in Appendix A. The only modification made was the addition of the specific quantitative measurement notations at the bottom third of the page due to the large amount of quantitative data collection I performed during both trials. The original worksheet prototype was utilized in Cleveland, Ohio, modified after returning, and subsequently the worksheet shown in Appendix A was implemented in Washington, D.C. In addition to completing the worksheet for each individual, I also logged the results in an analytical notebook, which kept data in duplicate. In this notebook I labeled the top columns "\#1" through "\#6" representing the six features to be observed, and the museum's log number, representing each individual, became the individual rows. The sex characteristic was logged as either " $\mathrm{M}$ " representing male or " $F$ " representing female. In addition to the six (6) distinctive visual traits recorded as either male or female, the presence of a septal aperture was also documented. Other distinguishing characteristics such as pathology, unusual features, or the general observation of "robust" or "gracile" were noted for each bone element.

\section{- Observation Techniques}

Trochlear constriction (Figure 1), trochlear extension (Figure 2), and olecranon fossa shape (Figure 3): In observing these traits, all located on the 
posterior surface of the humerus, I would set the anterior side of the bone on the foam-covered velvet and look directly down on the feature at a ninety-degree angle. This technique afforded me the best and most unobstructed view of the features.

Angle of the medial epicondyle (Figure 4): To examine the angle of the medial epicondyle, I was required to crouch down and observe the medial epicondyle at the "table top" level, by aligning my view with the level surface of the table. This enabled the observation of the angle as parallel to the table top (considered a male trait) or distinctly raised from the linear profile of the table top (considered a female trait).

Trochlear symmetry (Figure 5): observation of this trait required me to place the bone on its lateral edge and observe the medial surface of the distal humerus. When doing this, most often the bone will balance itself on its lateral surface when gently placed into position; the spur of the lateral epicondyle and the shape of the greater tubercle allow this balancing execution. To get a better "feel" for this feature, I took my two index fingers and placed them on either side of the medial epicondyle, and felt the amount of trochlear bone surface on each finger. If the amount felt (and appeared) almost equal, as if the medial epicondyle was placed in the center of the trochlear profile, this was determined a male trait. If my right index finger (the finger placed on the posterior portion of the trochlea if referring to a left humerus) slipped from the trochlear surface due to the lack of sufficient bone surface, this characteristic was considered "anterior asymmetry", or 
the observation of a greater amount of trochlear surface towards the posterior direction of the bone.

Distal spool curvature (Figure 6): to observe this feature successfully, I again was required to view the distal end while bending or crouching to align one's eyes to the tabletop level. The bone was kept in the same position as in the last feature, balanced on its lateral surface by the spur of the lateral epicondyle and the greater tubercle. Determination of sex was made while gauging the amount of curve (or lack thereof) compared to the horizontal surface of the tabletop.

I could complete the visual analysis of each bone usually within approximately 3 minutes, sometimes much more rapidly. An ambiguous or difficult trait would take additional time to determine, but I completed almost all humeral elements within 3 to 5 minutes. When I added the quantitative technique of measuring certain features, however, my analysis could take as long at 8 minutes as a time. The reasoning behind collecting measurements while doing a qualitative analysis was twofold. First, I wanted to take a random sampling of measurements to determine if in fact quantitative analysis would be a consistently better indicator of sex than the qualitative analysis I was performing, based on my own metric data. When some humeri were determined to be quite ambiguous in their visual characteristics, I completed measurements on those humeri to determine if quantitative techniques could classify an individual as male or female accurately where visual characteristics could not. All humeri considered too ambiguous to accurately sex with qualitative analysis were measured in order to 
attempt an accurate sex determination based on past quantitative methods (France 1988, Bass 1995).

\section{- Photography}

Over 300 photographs were created between both the Cleveland Museum of Natural History collection and the Smithsonian collections. Distal humeri that exhibited unambiguous and distinctive features for either male morphology or female morphology were chosen for photographic documentation. A set of three (3) clearly identifiable humeri were placed on black velvet, and the set of humeri were photographed to highlight the unique male or female characteristics of trochlear constriction, trochlear symmetry, olecranon fossa shape, the angle of the medial epicondyle, medial epicondylar symmetry, and distal spool curvature. Lighting was adjusted to adapt to the camera's abilities and limitations and to highlight certain features. This process was repeated with a series of four (4) bones photographed together, two (2) bones photographed together, and then photography of single distal humeral elements and their distinguishing characteristics. A photographic log was created for future reference. 